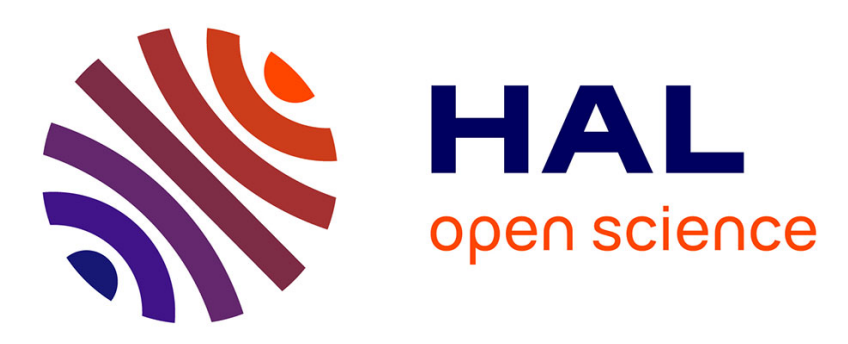

\title{
A computational approach to design new tests for viscoplasticity characterization at high strain-rates
}

Pascal Bouda, Bertrand Langrand, Delphine Notta-Cuvier, Eric Markiewicz, Fabrice Pierron

\section{- To cite this version:}

Pascal Bouda, Bertrand Langrand, Delphine Notta-Cuvier, Eric Markiewicz, Fabrice Pierron. A computational approach to design new tests for viscoplasticity characterization at high strain-rates. Computational Mechanics, 2019, 64, pp.1639-1654. 10.1007/s00466-019-01742-y . hal-02293111

\section{HAL Id: hal-02293111 \\ https://hal.science/hal-02293111}

Submitted on 20 Sep 2019

HAL is a multi-disciplinary open access archive for the deposit and dissemination of scientific research documents, whether they are published or not. The documents may come from teaching and research institutions in France or abroad, or from public or private research centers.
L'archive ouverte pluridisciplinaire HAL, est destinée au dépôt et à la diffusion de documents scientifiques de niveau recherche, publiés ou non, émanant des établissements d'enseignement et de recherche français ou étrangers, des laboratoires publics ou privés. 


\title{
A computational approach to design new tests for viscoplasticity characterization at high strain-rates
}

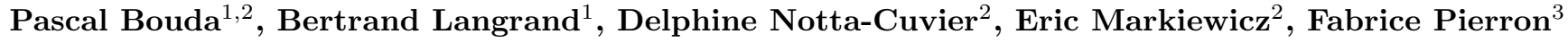 \\ 1 DMAS, ONERA, F-59014 Lille, France \\ 2 LAMIH UMR CNRS 8201, UPHF Le Mont Houy, F-59313 Valenciennes Cedex 9, France \\ 3 Faculty of Engineering and the Environment, University of Southampton, Highfield SO171BJ, UK
}

Received: date / Revised version: date

\begin{abstract}
Rate-dependent behaviour characterization of metals at high strain rate remains challenging mainly because of the strong hypotheses when tests are processed with statically determinate approaches. As a non-standard methodology, Image-Based Inertial Impact (IBII) test has been proposed to take advantage of the dynamic Virtual Fields Method (VFM) which enables the identification of constitutive parameters with strain and acceleration fields. However, most of the test parameters (e.g. projectile velocity, specimen geometry) are not constrained. Therefore, an FE-based approach is addressed to optimize the identification over a wide range of strain and strain-rate, according to two design criteria: (1) - the characterized viscoplastic spectra (2) - the identifiability of the parameters. Whereas the first criterion is assessed by processing the FEA simulations, the second is rated extracting material parameters using synthetic images to input the VFM. Finally, uncertainties regarding the identification of material constants are quantified for each IBII test configuration and different camera performances.
\end{abstract}

\section{Introduction}

Material dynamic behaviour characterization is often performed through standard tests. Their setup (possibly their processing) is often based on standards which have been initially defined for quasi-static tests. For instance, the standard ASTM E8-04 [1] reports a methodology for uniaxial tension testing of metallic materials. It relies on a statically determinate approach implying strong hypotheses on experimental test conditions. Thus, analysis assumes homogeneous small level of strain and strain rate over the specimen gauge length. Then, considering that the longitudinal nominal stress is proportional to load measured from test machine load cell, materials parameters can be extracted from the uniaxial stress-strain curve. However, the extension of such standards to high strain-rate testing is not straightforward because of the difficulty to achieve homogeneous mechanical fields, in particular the strain-rate [2]. For rate-dependent materials, the test campaign itself is more challenging because a high number of experiments is necessary with these approaches to characterize the behaviour over large spectra of strain and strain rate.

Owing to the dramatic improvements in imaging technology, it is now possible to measure kinematic fields at a large number of points over specimen surface (often referred to as "full-field measurements"). This is now also possible at high frame rates thanks to the growing technological developments in high speed cameras. Then, several inverse methods have been developed for identification of material parameters from full-field measurements. An overview of 
these methods is beyond the scope of this paper, but detailed reviews can be found in the literature, including for high strain-rate applications [2-4]. Among the available inverse methods, the Virtual Fields Method (VFM) can be used to identify material constitutive parameters from measurement of strain fields and either external forces [5] or acceleration fields [6]. The first approach was used to characterize viscoplastic Johnson-Cook (JC) model for titanium [7] and copper [8] alloys at moderately high strain rates (up to about $100 \mathrm{~s}^{-1}$ ) because inertia effects were not taken into account. The available literature about VFM with inertia effects for viscoplasticity characterization [9, 10] extends a study based on a bending test [11]. However, the specimen underwent small deformations only (0.02 at best) because the test configuration was set up without preliminary analyses. Therefore, the material behaviour was characterized on a limited viscoplastic spectra.

Image-Based Inertial Impact (IBII) tests have been recently proposed to take advantage of the VFM for material behaviour characterization at high strain rate [12] (up to about $1000 \mathrm{~s}^{-1}$ ). It consists in a thin-plate specimen hit by a cylindrical projectile on its edge at several tens of meters per second, while the opposite edge is free to move, this leading to a longitudinal rigid body motion. Using this experimental setup, elastic [12] and elasto-plastic [13] constitutive models have been identified. To extend the aforementioned tests to rate-dependent material behaviour, one needs to understand the influence of several tests parameters such as projectile velocity and geometry, specimen geometry and metrological features like camera specifications and full-field measurements regularization parameters. Indeed, these parameters all influence the resulting identification and it is essential to set them using a rational approach.

In this paper, we propose a computational approach to optimize test configurations, in terms of aforementioned parameters, for dynamic behaviour characterization of metals ( $\dot{\varepsilon}$ up to $10^{4} \mathrm{~s}^{-1}$ ), using IBII tests for the extraction of viscoplastic constitutive parameters. Finite Element Analysis (FEA) is used to analyze plastic strain and plastic strainrate spectra generated in the specimen for each configuration of testing and measurement parameters. Test parameters that lead to the richer spectrum for characterization of viscoplasticity are thus selected. The influence of experimental biases on material parameters identification is also studied. This is performed using synthetic images encoding FE simulated deformations, leading to realistic simulations of the complete measurement and identification chain. Owing to this procedure, IBII test configurations (including VFM identification) are optimized for JC viscoplastic parameters identification, taking into account the current limitations of measurement devices.

\section{Johnson-Cook model for rate-dependent elasto-plastic material behaviour}

Many constitutive models exists in the literature to describe the mechanical behaviour of metals, from early stages of loading until failure. For isotropic behaviour, constitutive models are generally based on von Mises (i.e. $J_{2}$-plasticity) theory, in conjunction with a hardening law which governs the evolution of the yield stress $\sigma_{y}$ [14] during loading. In absence of kinematic hardening and damage, $\sigma_{y}$ is then generally driven by temperature $T$, cumulated plastic strain $p$ and possibly by cumulated plastic strain-rate $\dot{p}$. For rate-dependent behaviour characterisation, some of the existing models have a physical background (e.g. Zerilli-Amstrong [15] or Rusinek-Klepazcko [16]), like dislocation mechanics and thermodynamics. However, they often involve a significant number of material parameters, hence are challenging to identify. Furthermore, their implementation in FEA codes is difficult because of the mathematical expression of their constitutive equations. By contrast, several phenomenological models (such as Cowper-Symonds [17], JohnsonCook [18] or Jones [19]) have been developed as a compromise between computing efficiency and representation of physical phenomena. The Johnson-Cook (JC) model is the most used in FEA. Its semi-empirical flow rule (Eqn. 1) 
can be split into three parts: a "static" term, $\sigma^{\text {stat }}$, similar to the Ludwik rate-independent isotropic hardening [14], a "dynamic" correction term, $\sigma^{\text {visc }}$, activated if the equivalent strain-rate $\dot{p}$ reaches a threshold value $\dot{\varepsilon}_{e q, 0}$, and a third term, $\sigma^{\text {th }}$, for thermal softening.

$$
\sigma_{y}=\underbrace{\left[\sigma_{0}+K p^{n}\right]}_{\sigma^{\text {stat }}} \underbrace{\left[1+M \ln \left(\frac{\dot{p}}{\dot{\varepsilon}_{e q, 0}}\right)\right]}_{\sigma^{\text {visc }}} \underbrace{\left[1-\left(\frac{T-T_{0}}{T_{f}-T_{0}}\right)^{m}\right]}_{\sigma^{\text {th }}}
$$

The JC model has been used to describe the behaviour of various metals such as iron [18, 20], copper [18,20], steels [18, 20-23] or lightweight alloys [24-26]. In practice, if neglecting thermal softening, the identification of JC constitutive parameters includes two steps which rely on statically determinate approaches. First, quasi-static tests are performed to identify the material parameters driving $\sigma^{\text {stat }}$. Then, dynamic tests are performed to extract the parameters driving $\sigma^{v i s c}$ using an high-speed hydraulic machine or/and Split Hopkinson Bar (SHB) apparatus [27,28] for instance. Finally, the combination of tests at low, moderate and high strain rate enables the characterization of material behaviour over a large range of strain rate. However, numerous tests are necessary because of the assumption of constant and homogeneous strain rate fields for each specimen.

To drastically reduce the test campaign, one solution would consist in generating heterogeneous strain and strain rate fields on a single specimen. Then, it would be necessary to process the test results with a statically undeterminate approach, i.e. method where no (or a few) assumption is made on tests conditions and nature of mechanical fields. For material behaviour characterization at high strain rate, Ultra-High-Speed (UHS) imaging devices are continuously being improved, so that full-field measurement techniques enable the tracking of these heterogeneous mechanical fields. The next section presents the VFM and the grid method which are used in this study for viscoplasticity characterization.

\section{VFM and grid method for dynamic behaviour characterization}

\subsection{The Virtual Fields Method (VFM)}

The Virtual Fields Method (VFM) [5] is based on the Principle of Virtual Work (PVW). For small perturbations, it can be expressed as follows:

$$
\begin{aligned}
-\int_{V} \underline{\underline{\sigma}}(\underline{X}): \underline{\underline{\varepsilon}}^{*} d V+\int_{\partial V} \underline{t} \cdot \underline{u}^{*} d S+\int_{V} \underline{f} \cdot \underline{u}^{*} d V & =\int_{V} \rho \underline{\gamma} \cdot \underline{u}^{*} d V \\
W_{i n t}^{*}(\underline{X})+W_{e x t}^{*}+ & =W_{a c c}^{*}
\end{aligned}
$$

where $V$ denotes the material volume, $\underline{\underline{\sigma}}$ the Cauchy stress tensor computed from full-field strain measurements and constitutive laws using a set of material parameters $\underline{X} \cdot \underline{u}^{*}$ is the virtual displacement field, $\underline{\varepsilon}^{*}$ the virtual strain tensor such as $\underline{\varepsilon}^{*}=\frac{1}{2}\left[\nabla \underline{u}^{*}+\left(\nabla \underline{u}^{*}\right)^{T}\right], \underline{t}$ the traction vector, $\underline{f}$ the body loads, $\rho$ the material density and $\underline{\gamma}$ the acceleration field. $W_{i n t}^{*}$ is the only term that depends on material parameters $\underline{X}$. $W_{\text {ext }}^{*}$ is the external virtual work which stands for the contribution of the external loads during the test. $W_{a c c}^{*}$ is the contribution of acceleration which is also representative of inertia effects. As external loads are difficult to measure in transient dynamic tests, it is convenient to zero the virtual displacement field $\underline{u}^{*}$ at the loaded surfaces. In absence of body loads, the PVW is then expressed by Eqn. 3 .

$$
\begin{aligned}
\int_{V} \underline{\underline{\sigma}}[\underline{X}]: \underline{\underline{\varepsilon}}^{*} d V+\int_{V} \rho \underline{\gamma} \cdot \underline{u}^{*} d V & =0 \\
-\quad W_{i n t}^{*}(\underline{X})+W_{a c c}^{*} & =0
\end{aligned}
$$


In the general case, the identification of non-linear material constitutive models with the VFM relies on the minimization of a cost function, $\Phi$, describing a distance to equilibrium based on the PVW (Eqn. 2) [7,29-31]. This cost function $\Phi$ may be defined by Eqn. 4, where $N_{i}$ is the number of monitored load steps (i.e. in practice the number of measured displacement maps).

$$
\Phi[\underline{X}]=\sum_{i=1}^{N_{i}}\left[\frac{W_{a c c}^{*(i)}-W_{i n t}^{*(i)}[\underline{X}]}{W_{a c c}^{*(i)}}\right]^{2}
$$

The normalization by $W_{a c c}^{*}$ ensures that all load steps can contribute equally to the cost function throughout the test. If only surface data are available from field measurement, uniform strain distribution through the thickness needs to be assumed. Here, the mechanical fields will be supposed constant through the thickness using a plane stress assumption. So, the volume integral $V$ can be reduced to a surface integral $S$ over which mechanical fields are measured. As full-field measurement techniques provide discrete data which are constant over the $k$-th elementary surface, the virtual field $\underline{u}^{*}$ and the related virtual strain tensor $\underline{\underline{\varepsilon}}^{*}$ can be averaged over each data point surface $S(k)$ (denoted later $\bar{\varphi}^{S(k)}$ for any quantity $\left.\varphi\right)$. Then, Eqn. 3 can be written in discretized form as in Eqn. 5 at any stage of the measurement. Once the displacement maps are retrieved, numerical differentiation (single in space for $\underline{\underline{\varepsilon}}$ and double in time for $\underline{\gamma}$ ) enables the calculation of all virtual work components for a given set of material parameters $\underline{X}$.

$$
\sum_{k}^{N} S(k)\left[\underline{\underline{\sigma}}[\underline{X}](k):{\underline{\bar{\varepsilon}^{*}}}^{S(k)}\right]+\sum_{k}^{N} \rho(k) S(k)\left[\underline{\gamma(k)} \cdot{\underline{u^{*}}}^{S(k)}\right]=0
$$

The VFM has already been applied to characterize elastic $[6,13,32-35]$, elastoplastic $[13,36]$ and viscoplastic [9, 10] behaviour at high strain rate (up to $\propto 10^{3} s^{-1}$ ) using inertia as the load cell. In some cases, an SHB apparatus was used as a loading setup $[6,10,32,36]$ though it was not really suitable for the VFM. Indeed, the SHB apparatus has been refined over the years to minimize inertia effects whereas here, we rely on these inertia effects to produce the load information. Thus, a novel test (Image-Based Inertial Impact or IBII) was used [12,13,34,35] to take full advantage of inertia effects in the VFM. The experimental setup consists in a cylindrical projectile propelled towards a thin plate specimen eventually impacted on its edge (Fig. 1). The loading here is due to the propagation of the strain waves, generating high levels of acceleration which are beneficial to the VFM in dynamics.

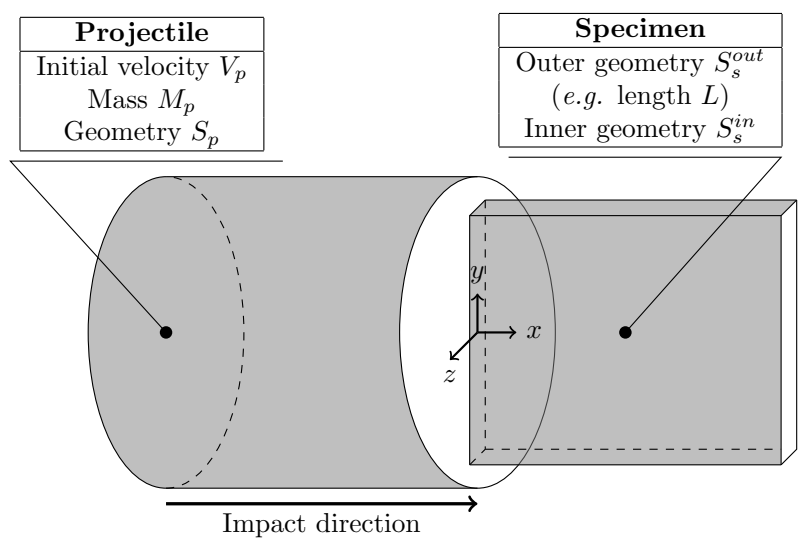

Fig. 1 Principle of the IBII test

Note that for IBII tests, $\underline{u}^{*}$ has to zero the contribution of impact force in the PVW (here at $x=0$, see Fig. 1). For instance, one like in Eqn. 6 may be incorporated in Eqn. 5, where $L$, the length of the specimen, is arbitrary fixed. 


$$
\mid \begin{array}{l|l}
u_{x}^{*}=x(x-L) & \varepsilon_{x x}^{*}=2 x-L \\
u_{y}^{*}=0 & \varepsilon_{y y}^{*}=0 \\
\varepsilon_{x y}^{*}=0
\end{array}
$$

\subsection{The grid method to extract strain and acceleration fields}

Among available techniques for full-field measurements, the grid method has been recognized as a good compromise between spatial resolution and displacement resolution, that favours providing rendering of strongly heterogeneous kinematic fields when using low resolution cameras [37]. The grid method relies on imaging a periodic grid bonded onto the specimen surface to register its deformation between a reference and deformed states.

To extract phase maps (which carry the displacement information) from greyscale images, a spectrum analysis is employed [38]. Here, Local Spectrum Analysis (LSA) was performed using a Windowed Discrete Fourier Transform (WDFT). As is customary with grid method, the phase is defined modulo $2 \pi$. Thus, an unwrapping algorithm [39] was implemented to correct the stacking of phase maps from spatial and temporal phase jumps. Temporal ones are caused by rigid body motion, and would strongly affect the calculation of the acceleration if ignored.

Displacement maps were then obtained with Eqn. 7, where $\underline{x}$ is the position vector, $\underline{u}$ the displacement vector, $\omega=\frac{2 \pi}{\Delta}$ the grid pulsation ( $\Delta$ the grid pitch), $\underline{\phi}^{R}$ and $\underline{\phi}^{D}$ reference and deformed phase maps respectively. Here, tested specimens undergo some rigid body motion owing to the nature of the test (impact with a free edge). Thus, the iterative approach described in [38] was used.

$$
\underline{u}[\underline{x}]=-\frac{1}{\omega}\left\{\underline{\phi}^{D}[\underline{x}+\underline{u}(\underline{x})]-\underline{\phi}^{R}[\underline{x}]\right\}
$$

As the WDFT analysis window spans two grid pitches, corrupted displacements are computed for the last grid pitch along the specimen edges. To replace them, Van Blitterswyk et al. [35] proposed to extrapolate data but so far, this method has been used on straight edges only. Here, the method was extended to handle the case of curved edges (e.g. circular), as present in specimens geometries used in the present work (see Sect. 5).

Strain and acceleration maps (which are input into the VFM) are deduced from displacement fields. As they are computed in a noisy environment in practice, robust differentiators have been designed in the frequency domain [40]. They enable both low pass filtering of displacement fields and derivatives calculation simultaneously. More precisely, a $n$-order differential of any quantity $\psi$ along $s$ is based on a centered stencil of neighbouring points (i.e. a weighted sum of discrete values of $\psi$ ). Finally $\left.\frac{\partial^{n} \psi}{\partial s^{n}}\right|_{m}$ is such as Eqn. 8, where $m$ is the step position, $N_{p}$ drives the stencil size, $\Delta s$ the pitch and $a_{i}(i \in \mathbb{Z})$ are the weighting coefficients. Their expression is determined enforcing an high-order tangency of the robust differentiator magnitude response at high frequencies. More details can be found in [40]. In practice, these derivatives are computed through convolution of fields maps with a sliding window based on coefficients $a_{i}$. Note that backward/frontward stencils can be considered to build formulæ for boundary points.

$$
\left.\frac{\partial^{n} \psi}{\partial s^{n}}\right|_{m}=\frac{1}{\Delta s^{n}}\left[a_{m} \psi_{m}+\sum_{i=1}^{N_{p}} a_{m-i} \psi_{m-i}-a_{m+i} \psi_{m+i}\right]
$$

\section{A numerical procedure to optimize IBII tests}

Up to now, elastic and elasto-plastic constitutive models have been characterized with IBII tests defined more or less intuitively. Yet, a robust design process is needed when aiming at characterizing more complex behaviour, such as ratedependent ones, from a single test. In this work, an FEA-based procedure is developed to optimize the characterization 
of viscoplasticity (JC model) using IBII tests and the VFM. It relies on the analysis of viscoplastic spectra driving the identification of the strain rate dependence and on the influence of experimental biases on material parameters identification. Contrary to an approach with real tests, the importance of all tests parameters can be studied thoroughly in quick succession. More precisely, the influence of impact velocity, specimen geometry and filtering parameters features will be analysed.

Assuming the knowledge of material behaviour, two design criteria have been selected to evaluate each test configuration:

- maximization of the range of validity of the identified viscoplastic model from single impact test: the configuration has therefore to span a viscoplastic spectrum $(p, \dot{p})$ as wide as possible;

- accuracy of material parameters identification with the VFM in realistic conditions: indeed, several parameters linked to the test itself (projectile and specimen geometries, impact velocity) or to the test processing (e.g. camera settings, full-field measurement technique, VFM toolchain features) influence the identification process, hence they must be taken into account in the design procedure of an optimized test.

In order to include the measurement toolchain in the design process, synthetic images were generated to simulate full-field measurements. This has already been addressed to quantify uncertainties in 2D-DIC [41] or to analyse the identification of elastic parameters with the VFM using quasi-static [42,43] and high strain-rate [34,44] tests. In those algorithms, an undeformed image of the specimen's region of interest (ROI) covered by a speckle (in case of DIC measurements) or a grid (grid method) is generated. Then sets of deformed images are built based on nodal displacements extracted from FEA, but without considering a fill factor, which is the fraction of the area of camera sensor which captures light intensity [45]. Here, we implement a mapping-based strategy which have been recently addressed [46]. However, the digitization step was updated to take into account the fill factor.

As the grid method is used here to track displacement fields, the texture of the initial image can be defined analytically [42-44]. Indeed, light intensity, $I$, at coordinates $(x, y)$ may be described by Eqn. 9 , where $\left(I_{0}, \gamma\right)$ are the mean intensity and the contrast respectively, and $\omega$ is the spatial pulsation of the grid $\left(\frac{2 \pi}{\Delta}\right)$. Note that if noise is taken into account, distributed random integers are added to pollute the encoded light intensity. Their definition domain is a fraction of the simulated camera dynamic range (a few percents).

$$
I(x, y)=I_{0}\{1+\gamma[\cos (\omega x)+\cos (\omega y)-|\cos (\omega x)-\cos (\omega y)|]\}
$$

In practice, the numerical simulations were carried out using the pre-processor of Cast3M® [47] to generate meshes and the explicit research code Europlexus@ [48] as FE solver. Continuum solid elements (8-node, fully integrated) were used to mesh the specimen and the mesh size $(0.5 \mathrm{~mm}$ outside of the refined areas) was deduced from a convergence analysis. Finally, a Python $\mathbb{R}$ toolchain handles both softwares and the data processing, from FEA results to the identification of the material parameters with the VFM (Fig. 2). The simulated mechanical fields were output at the surfaces nodes/elements, as full-field measurements provide displacement fields at the specimen surface only (see Fig. 3 in grey). The use of a 3D model will allow for the effect of possible lack of plane-stress condition at the hole/notch vicinity to be tested (Step 2.1 in Fig. 2).

\section{Application to the characterization of viscoplasticity}

The aforementioned methodology was applied to design IBII tests to characterize the behaviour of titanium alloy Ti6Al4V over a wide range of plastic strain and strain rate. This alloy is widely used in the aerospace industry owing 


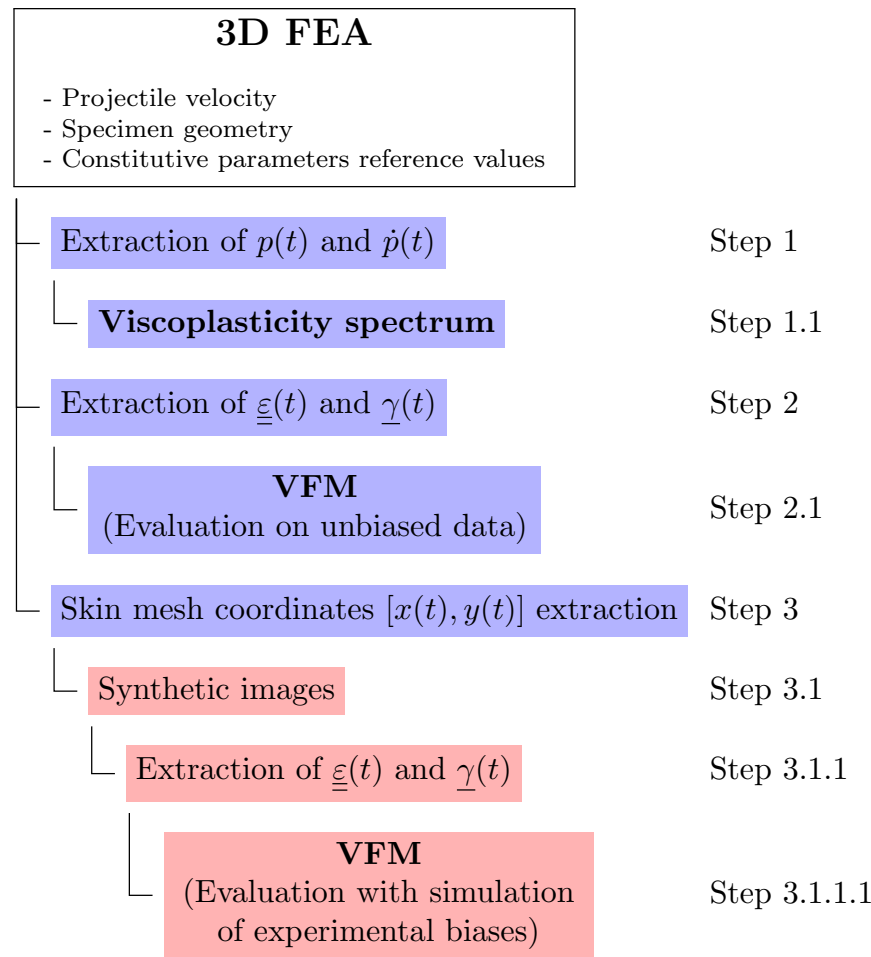

Fig. 2 FEA-based IBII tests toolchain analysis

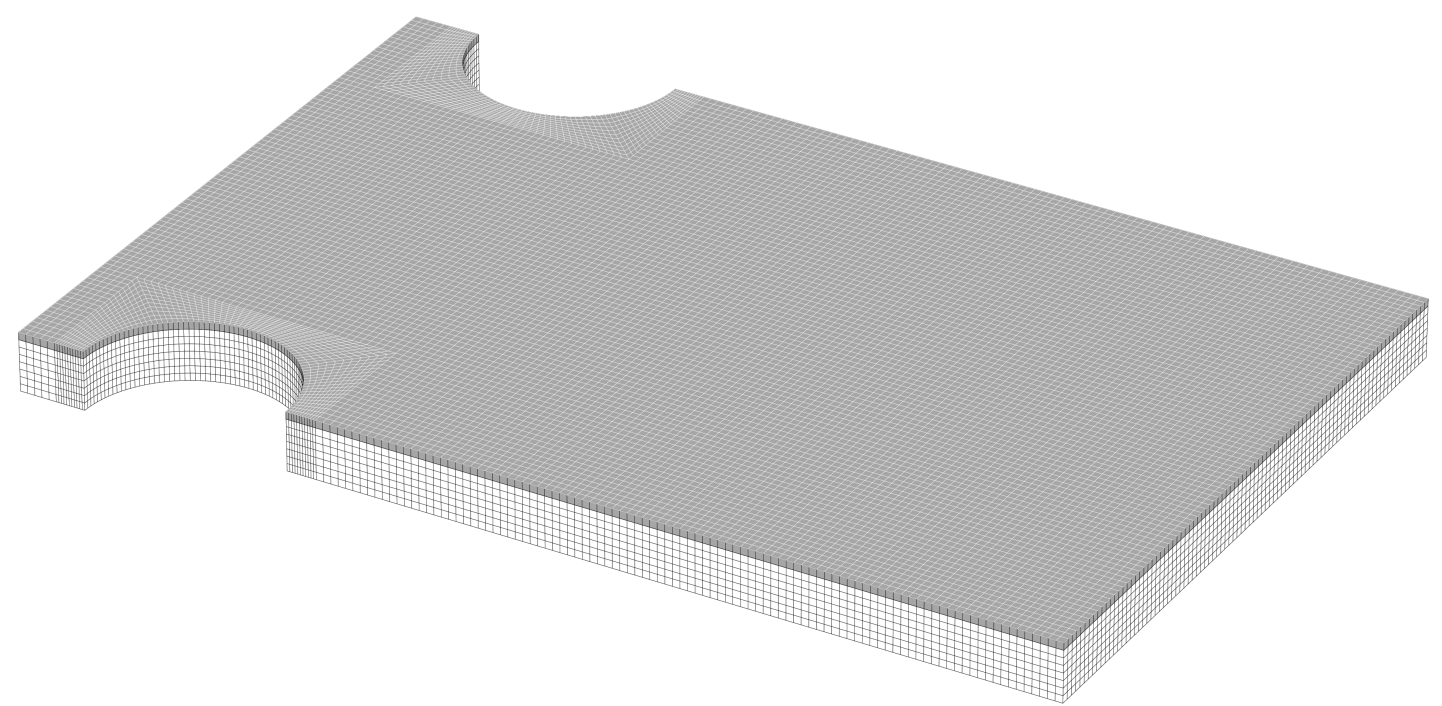

Fig. 3 Example of the 3D mesh of the double-notched specimen - mesh size: $0.5 \mathrm{~mm}$

to its high specific strength. In addition to Hooke's law for elastic linear behaviour, the JC flow rule defined by Eqn. 1 was considered to describe the viscoplastic flow. The temperature effects were neglected but could be introduced in the future if appropriate temperature measurements were available. Kinematic hardening and damage were not considered. Therefore, flow stress $\sigma_{y}$ varies with cumulated plastic strain $p$ and plastic strain-rate $\dot{p}$ only (Eqn. 10). The reference material parameters (Table 1) have been identified with standard techniques [7]. 


$$
\sigma_{y}=\mid \begin{array}{lr}
\sigma_{0}+K p^{n} & \text { if } \dot{p} \leq \dot{\varepsilon}_{e q, 0} \\
{\left[\sigma_{0}+K p^{n}\right]\left[1+M \ln \left(\frac{\dot{p}}{\dot{\varepsilon}_{e q, 0}}\right)\right]} & \text { if } \dot{p}>\dot{\varepsilon}_{e q, 0}
\end{array}
$$

Table 1 Ti6Al4V reference material constants for the Johnson-Cook's model [7]

\begin{tabular}{lc} 
Material constants & Value \\
\hline Young modulus, $E(\mathrm{MPa})$ & 114000 \\
Poisson ratio, $\nu(-)$ & 0.34 \\
Initial yield stress, $\sigma_{0}(\mathrm{MPa})$ & 973 \\
Isotropic hardening modulus, $K(\mathrm{MPa})$ & 560 \\
Isotropic hardening exponent, $n(-)$ & 0.562 \\
Viscoplastic parameter, $M(-)$ & 0.033 \\
Equivalent strain-rate threshold, $\dot{\varepsilon}_{e q, 0}^{p}\left(s^{-1}\right)$ & 1.29 \\
\hline
\end{tabular}

The cost function to be minimized through the VFM process (Eqn. 4) is built with the virtual field defined by Eqn. 6 (where $L$ is the specimen initial length) which remains constant whatever the time step $i$ because the PVW is always computed using initial coordinates of points of measurement. Finally, Eqn. 11 is obtained, where $\underline{X}$ is the set of material parameters, $N_{i}$ the number of synthetic images and $N_{p}$ the number of measurement points. To calculate stress fields from strains maps, a return mapping algorithm has been developed for the JC model, assuming a plane stress state. More information about its implementation can be found in the literature $[7,49,50]$.

$$
\Phi(\underline{X})=\sum_{i}^{N_{i}}\left[1+\frac{\sum_{k=1}^{N_{p}}\left[\sigma_{x x}(i, k, \underline{X}) \overline{2 x-L}^{S(k)}\right]}{\rho \sum_{k=1}^{N_{p}}\left[\gamma_{x}(i, k) \overline{x(x-L)}^{S(k)}\right]}\right]^{2}
$$

Inverse problems dealing with constitutive parameters identification often lead to the minimization of ill-conditioned cost-functions because of the imbalance in the sensitivities to the different material parameters, as in [36] for elastoplastic parameters. To avoid this problem, as a first step of development, monovariable cost-functions were considered. Thus, this study concentrates on the identification of JC viscoplastic parameter $M$ only. The hardening part $\sigma^{\text {stat }}$ of the model has been supposed known; in practice, it can be identified using a standard tensile test performed at quasi-static strain rate. The minimization of $\Phi$ (Eqn. 11) w.r.t the set of material parameters $\underline{X}$ (here reduced to the JC viscoplastic parameter $M$ ) was carried out with the Nelder-Mead algorithm. The latter is suitable for cost-efficient non-linear minimization when derivatives do not have to be calculated.

Table 2 reports impact tests configurations, assuming notations of Fig. 1. The projectile is a steel cylinder $(\mathrm{E}=200 \mathrm{GPa}, \nu=0.3)$ fired at $V_{p}=70 \mathrm{~m} \cdot \mathrm{s}^{-1}$. Impact conditions must be set to enable viscoplasticity characterization, i.e. to ensure the propagation of (visco)plastic waves in all proposed specimens. Yet, as the studied material has a high initial yield stress (973 MPa - Table 1), it may be necessary to "machine" holes or notches to the initially designed rectangular specimen to ensure high levels of plastic strain and plastic strain rate and identify the JC model over a large range of strain and strain rate. Three specimen geometries have been analysed for IBII tests impact conditions (Fig. 4): a rectangular one (Specimen 0) as already used for elastic(-plastic) behaviour characterization in the literature $[12,13,34,35]$, a single-holed one (Specimen 1) and a double-notched one (Specimen 2) with the notch 
location at the same x-coordinate. The latter has been chosen among hand-picked positions to favour (visco)plastic waves propagation. Note that the same amount of material has been "machined" so Specimens 1 and 2 have the same mass. As all specimens have the same length, impact duration - that corresponds to the back-and-forth travelling time of a longitudinal elastic wave through the specimens - is always of about $25 \mu$ s, whichever the impact velocity.

Table 2 Test configuration

\begin{tabular}{lc} 
Projectile features & \\
\hline Length $L_{p}(\mathrm{~mm})$ & 70 \\
Base radius $R_{p}(\mathrm{~mm})$ & 24.5 \\
\hline Specimen features & 70 \\
\hline Length $L_{s}(\mathrm{~mm})$ & 45 \\
Width $(\mathrm{mm}) D_{s}$ & 4 \\
Thickness $H_{s}(\mathrm{~mm})$ & $\{7,11.5\}$ \\
Hole/Notch radii $R_{s}$ and & \\
x-coordinate $(\mathrm{mm})$ - optional & \\
\hline
\end{tabular}

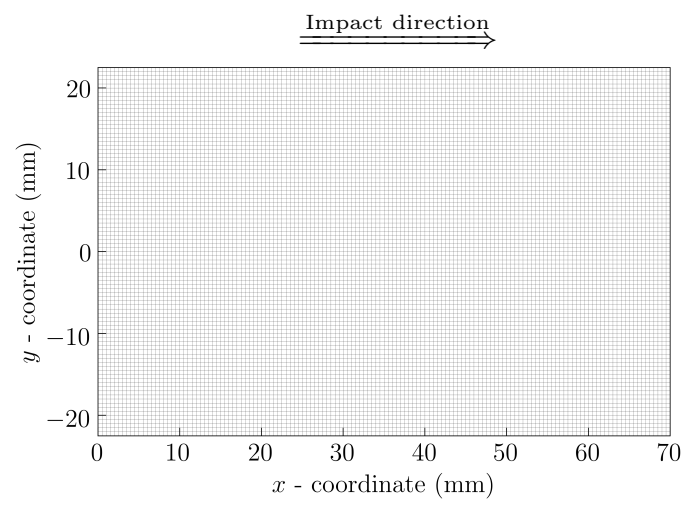

(a)

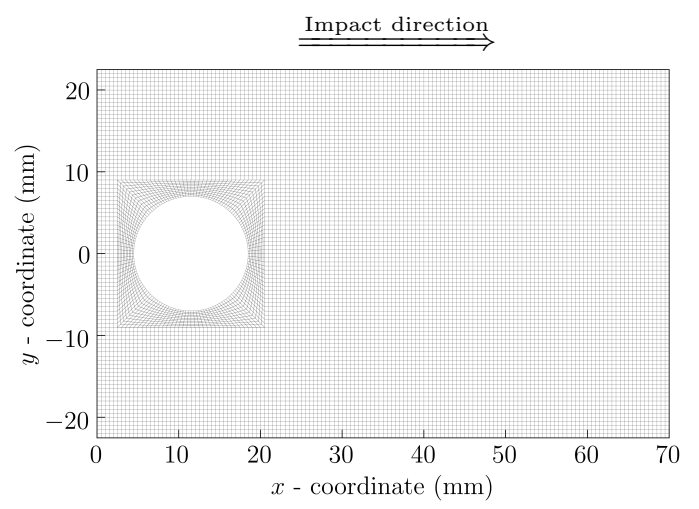

(b)

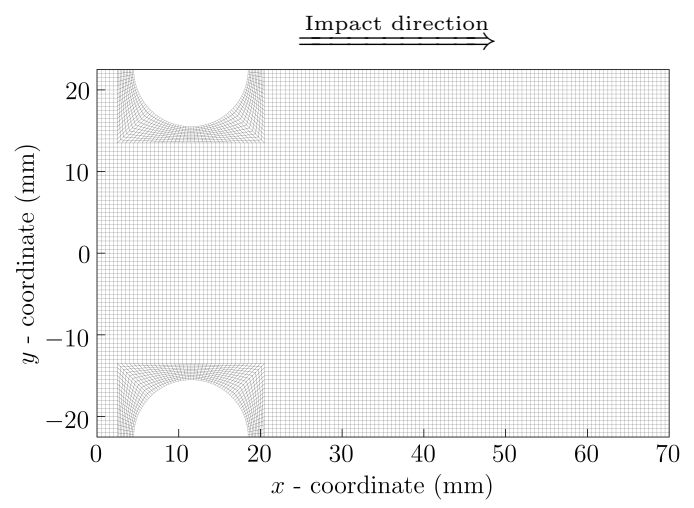

(c)

Fig. 4 Specimen geometries - (a) Specimen 0 (rectangular) - (b) Specimen 1 (single-holed) - (c) Specimen 2 (double-notched)

\subsection{Evaluation of IBII test parameters through FEA data}

5.1.1 Influence of specimen geometry and impact velocity on viscoplastic spectra One design criterion for the IBII test is the content of the viscoplastic spectra. Here, these spectra are analyzed thanks to histograms which account for 
viscoplastic flow development during impact (see Fig. 2 - Step 1.1). All (visco)plastic states $(p, \dot{p})$ computed by FEA in the elements of the specimen upper layer are collected. As the mesh was refined around the stress concentrators, the contribution of each element to the counting is proportional to its surface. Thus, a viscoplastic state from a nominal element (i.e. one located in a non-refined zone) accounts for one whereas a state from a refined element accounts for the ratio between its area and the area of a nominal element. Fig. 5 shows the evolution of specimens viscoplastic spectra during impact. It can be observed that:

- all tests produced band-shaped viscoplastic spectra, i.e. higher (resp. lower) plastic strain occurred at higher (resp. lower) plastic strain rate, whichever the specimen geometry. This is due to the loading which is mainly unidirectional, though load path could be locally heterogeneous;

- the intermediate $(\mathrm{t} \approx 12 \mu \mathrm{s})$ and last $(\mathrm{t} \approx 25 \mu \mathrm{s})$ viscoplastic spectra are similar, i.e. viscoplastic flows have mostly expanded during early stages of wave propagation;

- the highest numbers of occurrences are observed for plastic strain rate about $\dot{p} \propto 10^{3} \mathrm{~s}^{-1}$. This is in good agreement with an approximation of the mean strain-rate as $\dot{\varepsilon} \propto V_{p} / L_{s}=10^{3} \mathrm{~s}^{-1}$. 
苛 急
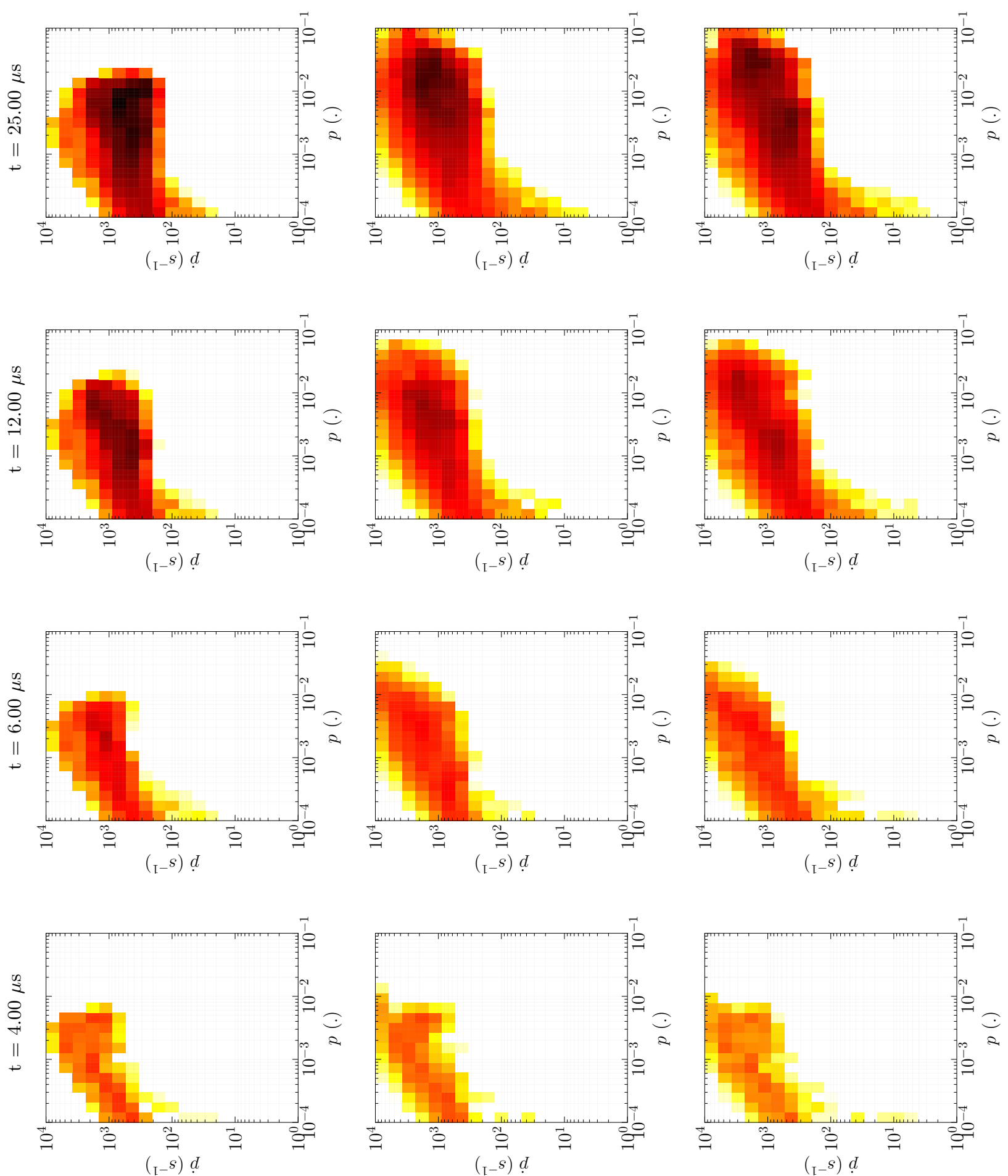

0 uəแ!̣วədS

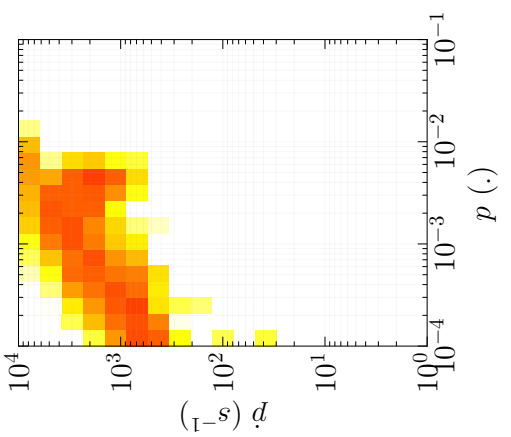

I иəụฺวdS

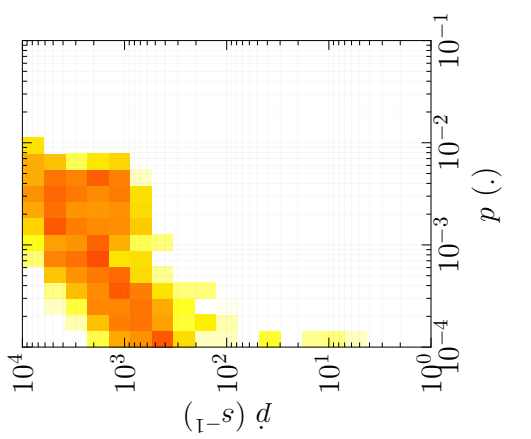

そ. иəụฺวədS 
One can also observe that geometrical discontinuities enrich the viscoplastic spectrum with a better coverage of the viscoplastic range (Fig. 6). Yet, presence of geometrical discontinuities do not strongly enhance large viscoplastic strains at low rate $\left(p>10^{-3}\right.$ and $\left.\dot{p}<10^{2} \mathrm{~s}^{-1}\right)$, so that less than a half of the global zone is covered. Furthermore, these large spectra are a sign of larger strain gradients. Their presence may jeopardize the identification if UHS camera temporal and spatial resolution are not sufficient to deal with them (see next sections).
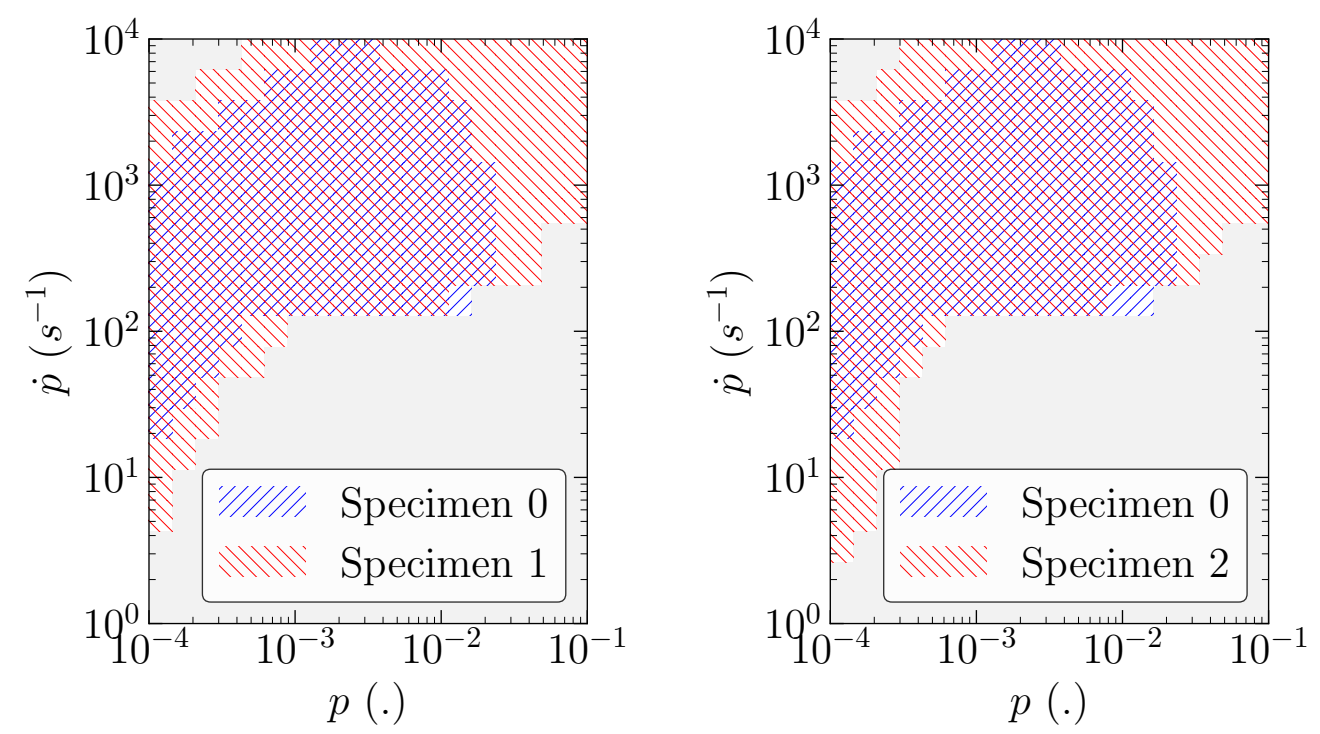

Fig. 6 Specimen-based histograms overlap

Another test parameter which may influence viscoplastic spectra is the projectile velocity. Thus, IBII tests simulations were carried out with the same test configuration (Table 2) except the projectile velocity that was set to $V_{p}=80 \mathrm{~m} \cdot \mathrm{s}^{-1}$, which corresponds to an increase of input kinetic energy of more than $30 \%$. As a first result, the peak value of $p$ increases (Fig. 7). This effect is more pronounced in Specimen 0 as it does not have the strain concentrators which mitigate the influence of increasing speed. Furthermore, plastic waves penetration seems to depend more on the specimen geometry than on projectile velocity, insofar as the penetration length decreases if there are geometrical discontinuities, whichever the value of $V_{p}$ (Fig. 7). More precisely, plastic waves do not penetrate behind holes/notches owing to the upstream wave reflections. By contrast, upper and lower boundary effects favour the penetration of plastic waves, hence the shape of its front end.

In a nutshell, those analyses highlight some advantages of holes/notches for viscoplastic flow expansion. For given impact conditions, their presence enhance the development of viscoplastic spectra which are suitable to characterize viscoplasticity over a wider range of strain and strain-rate. Moreover, projectile velocity is not as critical as one would have expected for the proposed configurations. However, this strengthens the idea that specimen design is a key point for IBII tests aiming at the extraction of constitutive parameters for a wide viscoplastic spectrum with a reduced number of tests. Finally, the present criterion (strain rate heterogeneity) does not tell anything about practical identifiability in real tests, this is investigated in the next section.

\subsubsection{Influence of temporal resolution and impact velocity on viscoplastic parameter extraction Extraction of vis-} coplastic parameters with the VFM requires the knowledge of several mechanical quantities computed from temporal and spatial differentiations of displacements to express cost-function $\Phi(\underline{X})$ (Eqn. 11) built from the reduced expression of the PVW (Eqn. 2). As already mentioned, in addition to strain and acceleration fields calculated from displacement 

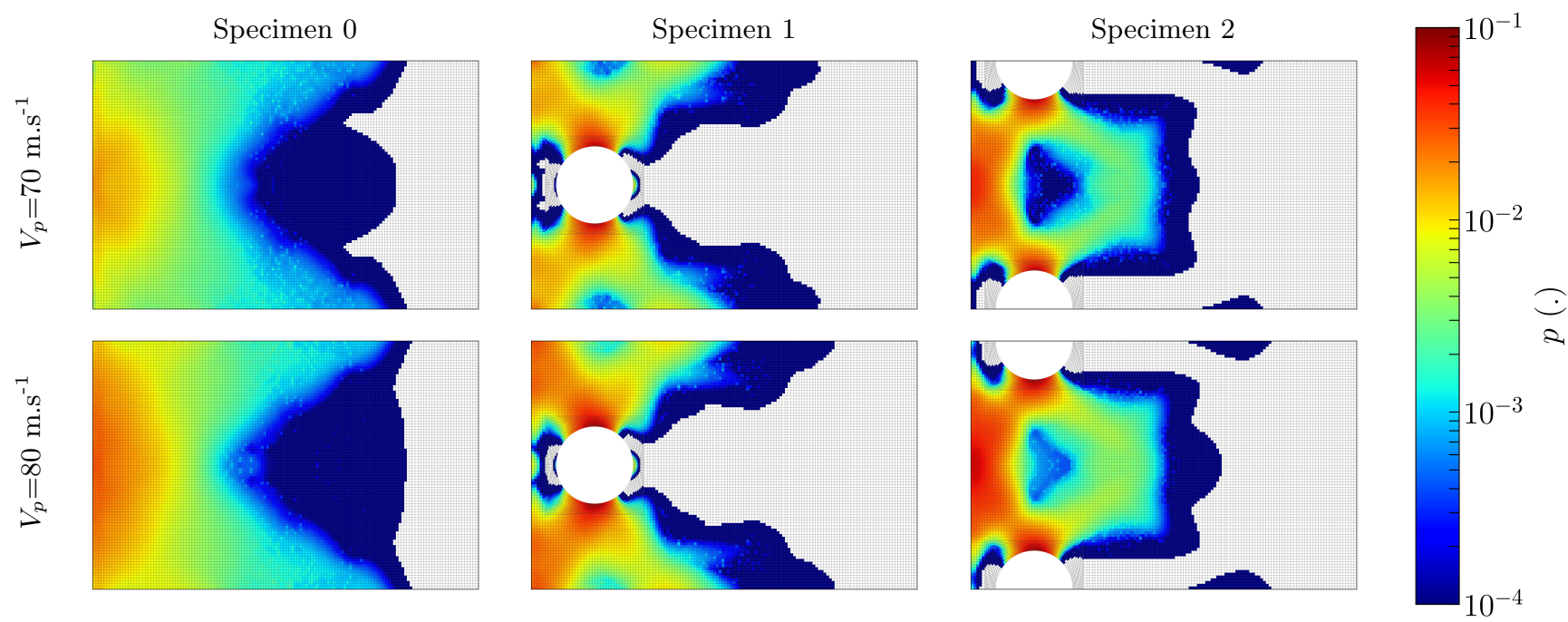

Fig. 7 Cumulated plastic strain $p$ thresholded at $10^{-4}-\mathrm{t}=12 \mu \mathrm{s}$

derivatives, a return mapping algorithm $[49,50]$ was implemented and validated to compute stress fields from strains with the JC model rate-dependent constitutive equations, assuming a plane stress state and using backward Euler method for time integration owing to its unconditional stability. However, this is a one step numerical scheme, hence integration highly depends on the size of the time increment, i.e. experimentally on the camera frame rate.

The VFM toolchain (enabling the identification of JC viscoplastic parameter $M$ ) was input with FEA strain and acceleration fields for various temporal resolution by undersampling FEA results, hence the simulation of different camera frame rates (see Fig. 2 - Step 2.1). This enables the quantification of systematic identification errors due to temporal resolution and plane stress assumption. Indeed, each 3D FE simulation - carried out with the suitable time step - can be undersampled to match a given frame rate, and only data on the specimen surface is processed. Each element of the top surface is equivalent to a measurement point, from which strain fields are computed by averaging Gauss points strain values within the element, and accelerations fields averaging nodal values at the surface of the element. Finally, the identified value of $M$ is compared to the reference value (input in the FE simulation) for the three considered specimen geometries and the two impact conditions, i.e. $V_{p}=70 \mathrm{~m} \cdot \mathrm{s}^{-1}$ (Fig. 8(a)) and $V_{p}=80 \mathrm{~m} . \mathrm{s}^{-1}$ (Fig. 8(b)).

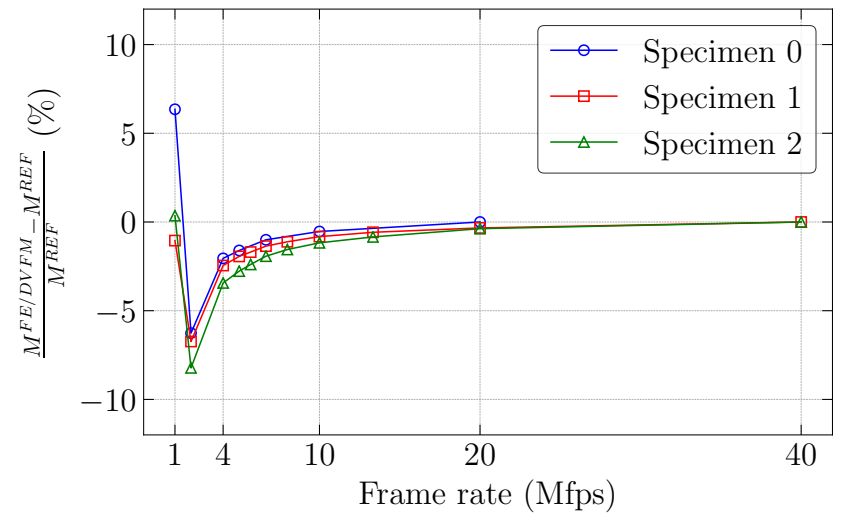

(a)

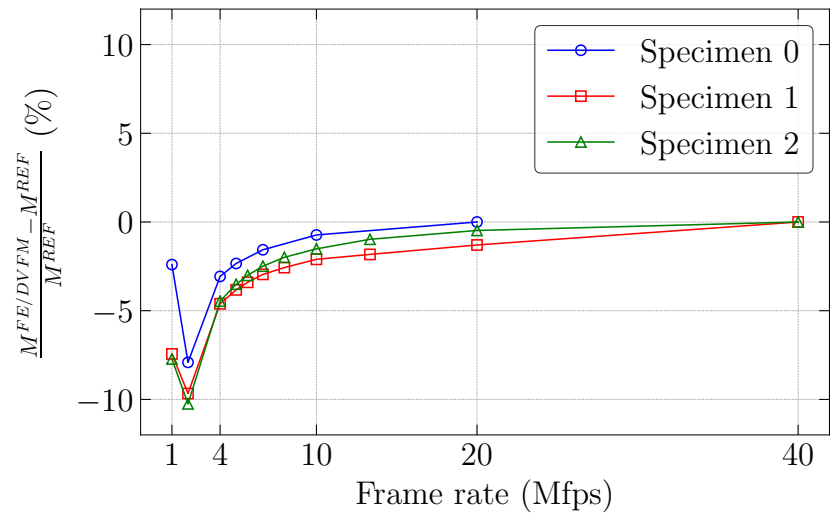

(b)

Fig. 8 VFM identification results for viscoplastic parameter $M$ from FEA data - (a) $V_{p}=70$ m.s s $^{-1}$ (b) $V_{p}=80 \mathrm{~m} . \mathrm{s}^{-1}$ 
For all IBII tests configurations, the identification converges towards the reference value (Fig. 8), when the simulated frame rate matches the FE time step (20 Mfps for Specimen 0 and 40 Mfps for Specimens 1 and 2). This demonstrates the relevancy of the plane stress assumption because JC viscoplastic parameter $M$ reference value is retrieved based on surface strain and acceleration fields only. However, the identification is not robust before a minimum temporal resolution of $4 \mathrm{Mfps}$ for both projectile velocities. Part of the systematic error is due to constitutive equation integration, whichever the specimen geometry. Furthermore, the identification of JC viscoplastic parameter $M$ is sensitive to the impact speed, as expected. Indeed, Fig. 8(a) exhibits a faster convergence towards the reference value if $V_{p}=70 \mathrm{~m} . \mathrm{s}^{-1}$. For $V_{p}=80 \mathrm{~m} \cdot \mathrm{s}^{-1}$ (Fig. 8(b)), $p$ and $\dot{p}$ temporal gradients are higher, hence an identification which is more sensitive to frame rate.

Now that systematic errors due to plane stress assumption and temporal resolution have been investigated based on FE data (e.g. underestimation of value of $M$ by $2 \%, 3.4 \%$ and $2.5 \%$ for Specimens 0,1 and 2 respectively at 4 Mfps and $V_{p}=70 \mathrm{~m} . \mathrm{s}^{-1}$ ), the synthetic image generator is used to study in more depth the effects of camera specifications (spatial and temporal resolution) and noise/regularization on the identification performance.

\subsection{Evaluation of IBII test parameters based on synthetic images}

Because of limited interframe time and spatial resolution, UHS cameras may provide data which are not accurate enough for material parameters identification under high strain rate loadings, in the presence of high spatial and temporal gradients of displacement and strain. Even if recent cameras offer better temporal resolution suitable for dynamic testings (at least $1 \mathrm{Mfps}$ ), spatial resolution often remains low (see the Shimadzu HPV-X®) properties in Table 3). In previous section, the minimum temporal resolution which is necessary for robust JC viscoplastic parameter for the proposed VFM toolchain was established at $4 \mathrm{Mfps}$. Yet, full-field measurements biases were not taken into account.

The aim of this section is to analyse for each proposed specimen the influence of camera temporal and spatial resolution for the identification of viscoplastic parameter $M$ (see Fig. 2 - Step 3.1.1.1). Therefore, synthetic images have been generated from simulated IBII tests with $V_{p}=70 \mathrm{~m} \cdot \mathrm{s}^{-1}$ for the three proposed specimens up to 10 Mfps. While the spatial resolution is directly taken into account by the digitization, temporal resolution is tuned by sub-sampling the time steps. Finally, the images are processed to identify parameter $M$ using the complete chain summarized in Fig. 9 .

Table 3 Initial camera settings (based on Shimadzu HPV-X specifications)

\begin{tabular}{lc}
\hline Resolution & $400 \times 250$ pixels \\
Interframe time & up to $0.2 \mu \mathrm{s}(5 \mathrm{Mfps})$ \\
Sensor & FTCMOS (on-chip storage) \\
Object pixel size & $0.182 \mathrm{~mm} /$ pixel \\
Dynamic range & 10 bits $(1024$ grey levels $)$ \\
\hline
\end{tabular}

The procedure can be applied to perfect or noisy images (Fig. 10). The latter are generated by adding random normally-distributed (over $1.5 \%$ of the camera dynamic range) integers to the noise-free greyscale images. The robust differentiators introduced in Sect. 3.2 are used on noisy images to provide some regularization. The stencil size, $N_{p}$ (Eqn. 8) is the regularization parameter. 


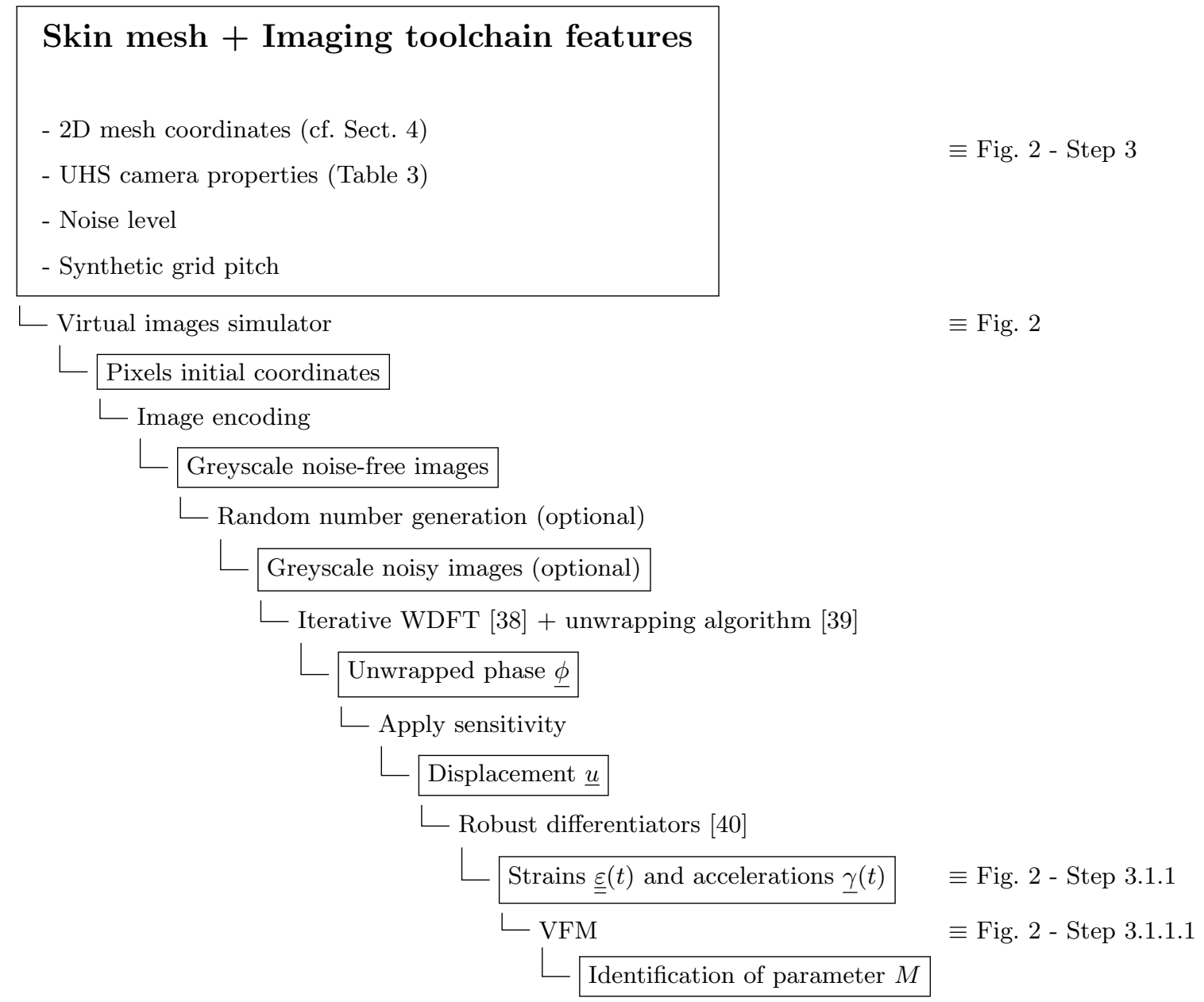

Fig. 9 Flowchart for VFM on synthetic images

For given camera properties, the regularization parameters will be selected as those minimizing the expanded uncertainty on the identified JC viscoplastic parameter $M$. The expanded uncertainty is defined here as $\epsilon^{\text {noise-free }}+$ $2 \sigma\left[\epsilon^{\text {noise }}\right]$, where $\epsilon^{\text {noise-free }}$ is the systematic error calculated based on noise-free images and $2 \sigma\left[\epsilon^{\text {noise }}\right]$ is the random error, computed here as the standard deviation of the distribution of identified $M$ for six sets of noisy images for each specimen geometry.

Fig. 11 maps the systematic error on parameter $M$ for the three specimen geometries imaged at 4 Mfps. For noise-free images, regularization reduces strain and acceleration peaks, so that the identification error increases with stencil size. The lowest error is obtained without regularization, corresponding to the use of central finite difference scheme to calculate strains and accelerations. As expected, notched and holed configurations (Specimen 1 and 2 ) are more sensitive to spatial regularization than Specimen 0, because of the presence of stronger spatial gradients. They seem slightly more sensitive to temporal regularization though this may not be significant.

Fig. 12 maps the expanded uncertainty in presence of noise, for the same identifications. It was impossible to identify a value for the $\mathrm{JC}$ viscoplastic parameter $M$ without temporal regularization. This was somewhat expected as acceleration fields require a double time-differentiation. Consequently, the set of optimal parameters is "shifted" from no regularization (in case of noise-free data) to both/either temporal and/or spatial regularization to a bit of temporal regularization (5 data points stencil). The mapping of standard deviation (Fig. 13) shows that its magnitude is hardly sensitive to regularization parameters, so that for a given set of temporal and spatial stencils, expanded uncertainty 


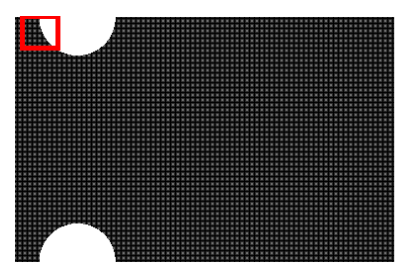

(a)

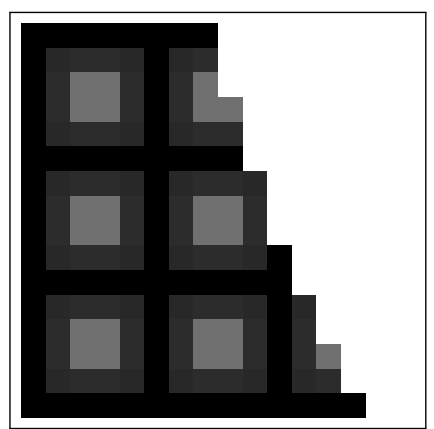

(b)

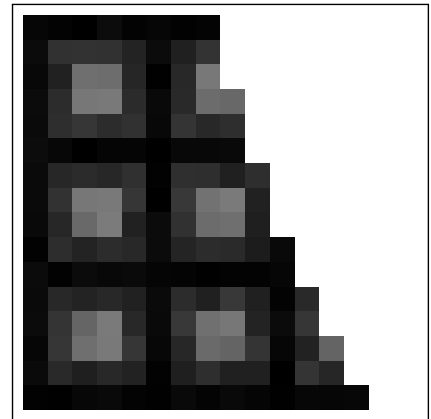

(c)

Fig. 10 Example of synthetic images processed by the grid method - (a) Full initial synthetic image (Specimen 2) - (b) Zoomed in view without noise - (b) Zoomed in view with noise

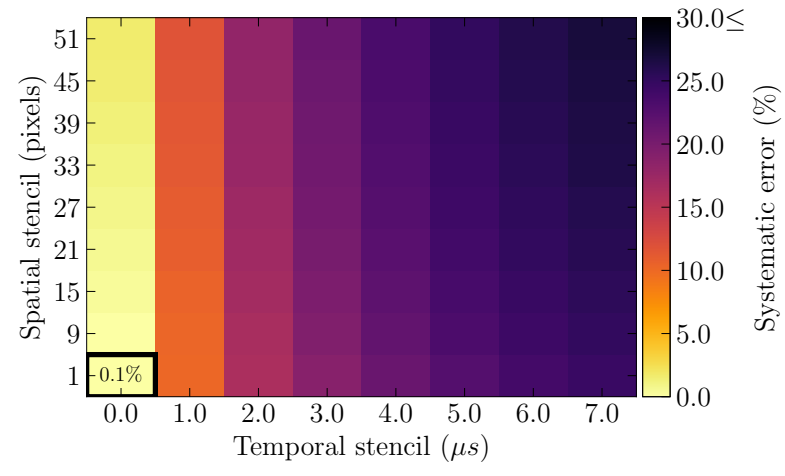

(a)

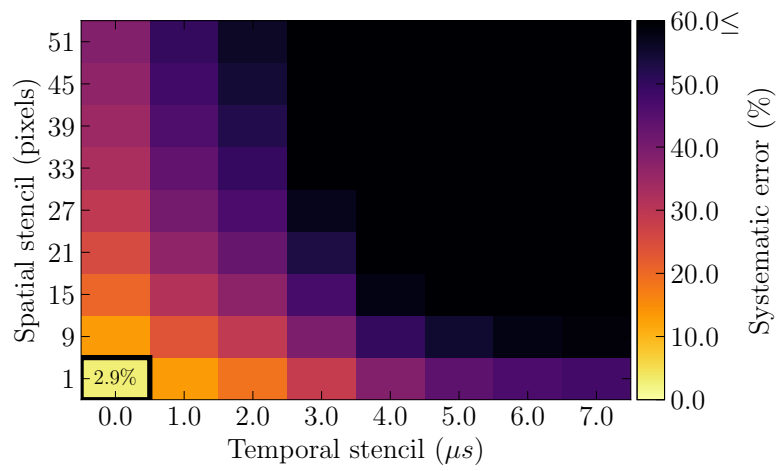

(b)

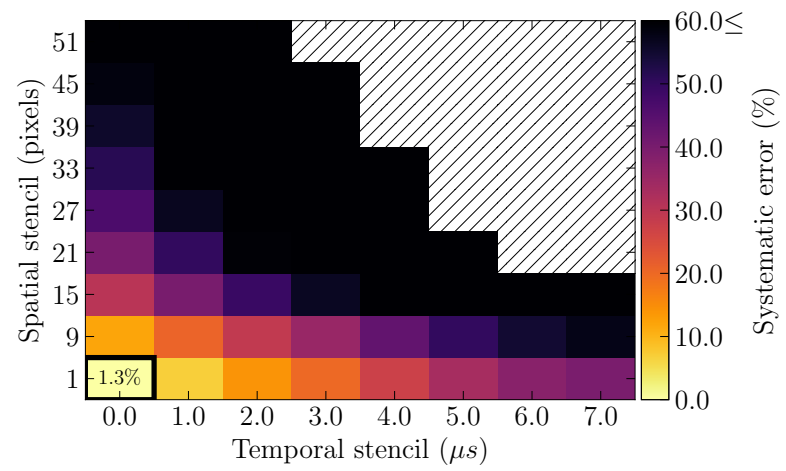

(c)

Fig. 11 Systematic error on parameter $M$ at $4 \mathrm{Mfps}$ and for $V_{p}=70 \mathrm{~m} \cdot \mathrm{s}^{-1}$ (unsuccessful identifications in hatched lines) - (a) Specimen 0 - (b) Specimen 1 - (b) Specimen 2

is mostly dominated by the systematic error rather than the random one.

Applying this methodology for different IBII test configurations and achievable frame rates enables uncertainty quantification with respect to regularization parameters. The results are reported in Fig. 14 with optimal regulariza- 


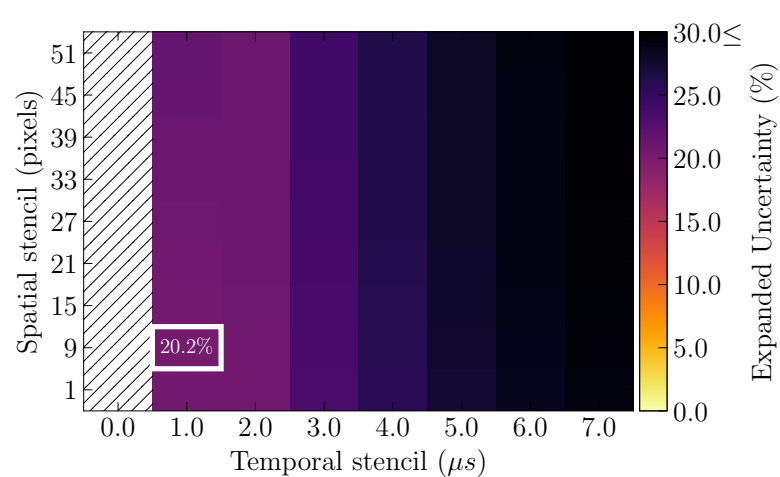

(a)

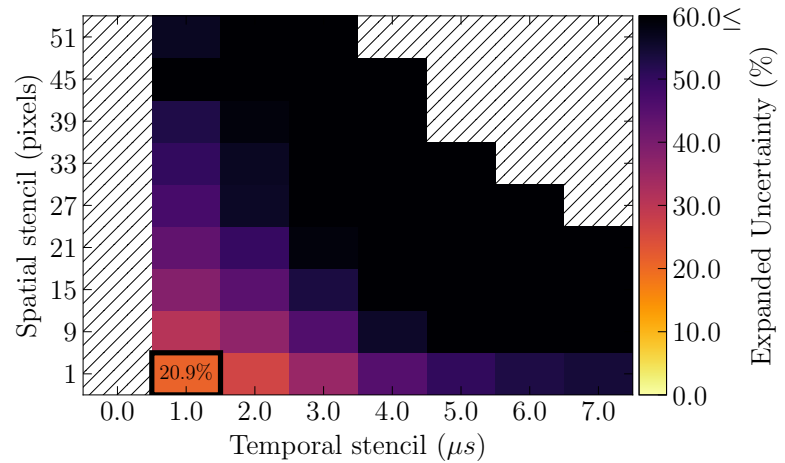

(b)

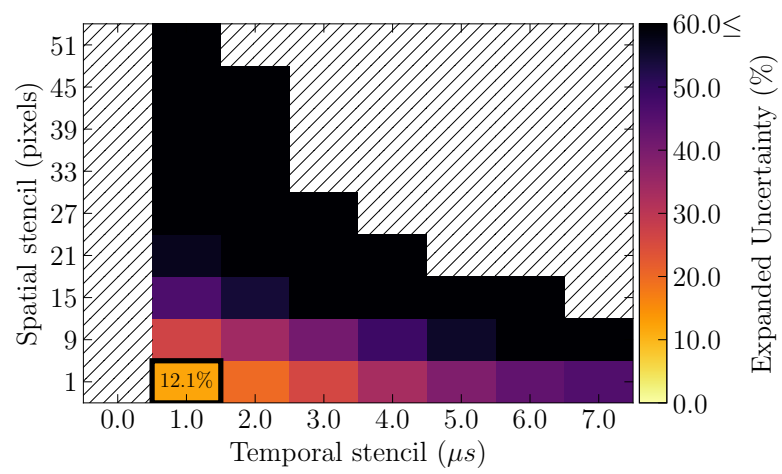

(c)

Fig. 12 Expanded uncertainty on parameter $M$ at $4 \mathrm{Mfps}$ and for $V_{p}=70 \mathrm{~m} \cdot \mathrm{s}^{-1}$ (unsuccessful identifications in hatched lines) (a) Specimen 0 - (b) Specimen 1 - (b) Specimen 2

tion parameters. At $4 \mathrm{Mfps}$, the identification error (systematic and random) is stabilized for all specimens as with FEA data (Fig. 8(a)).

To analyse the impact of the predicted expanded uncertainty of identified value of parameter $M$ on material behaviour, the return mapping algorithm has been input with FEA strains for each specimen and values of $M$ identified at $4 \mathrm{Mfps}$ for the same specimen. Fig. 15 shows the elements (pointed out by the dotted lines) that are monitored in each case. They have been chosen as critical zones over which viscoplastic parameter strongly influences the stress field. For those elements, the reconstructed curves (with the identified parameter) of $\sigma_{y}$ and $\dot{p}$ as a function of $p$ have been compared to ones extracted from FEA (Fig. 16).

It is worth noticing that the values of identified parameter lead to consistent results, though the better accuracy is for the notched specimen (Specimen 2) as expected according to the prediction of uncertainty. For this specimen, the yield stress computed by FEA almost lies within the uncertainty domain, whichever the cumulated plastic strain-rate (up to about $10^{4} \mathrm{~s}^{-1}$ ). However, the results are less satisfying for the two other specimens (Specimen 0 and 1 ). This was expected according to the higher expanded uncertainty.

This work on synthetic images reveals the advantages and drawbacks on the proposed IBII test configurations and VFM toolchain in more realistic conditions. The low systematic error on noise-free synthetic images validates full-field measurement processing, notably the plane stress assumption and data extrapolation due to information lost on specimen edges. This is particularly important as for the holed and notched specimens, large strains and strain rates 


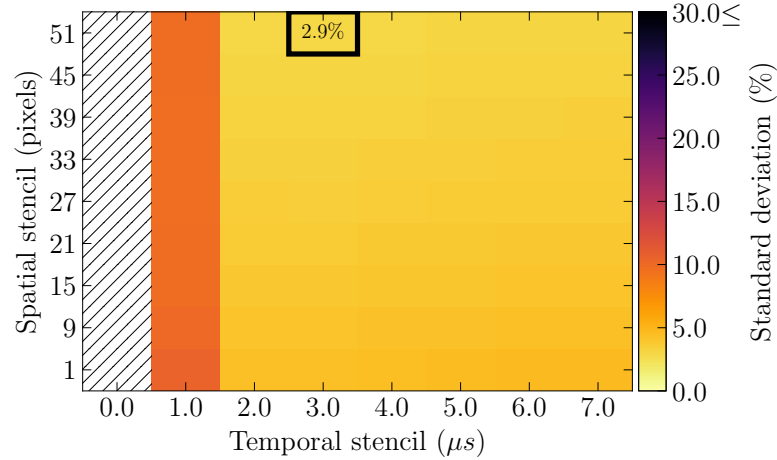

(a)

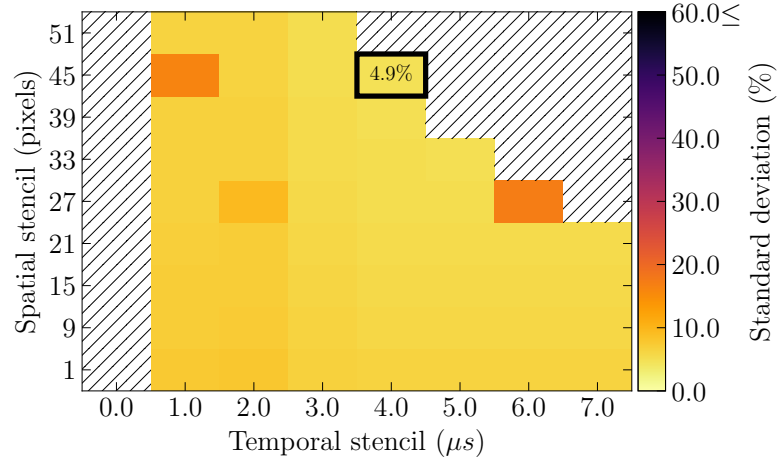

(b)

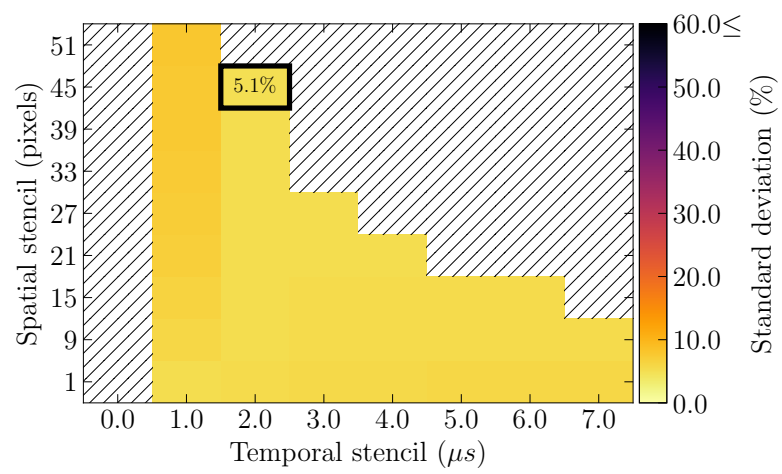

(c)

Fig. 13 Standard deviation on parameter $M$ at $4 \mathrm{Mfps}$ and for $V_{p}=70 \mathrm{~m} \cdot \mathrm{s}^{-1}$ (unsuccessful identifications in hatched lines) - (a) Specimen 0 - (b) Specimen 1 - (b) Specimen 2

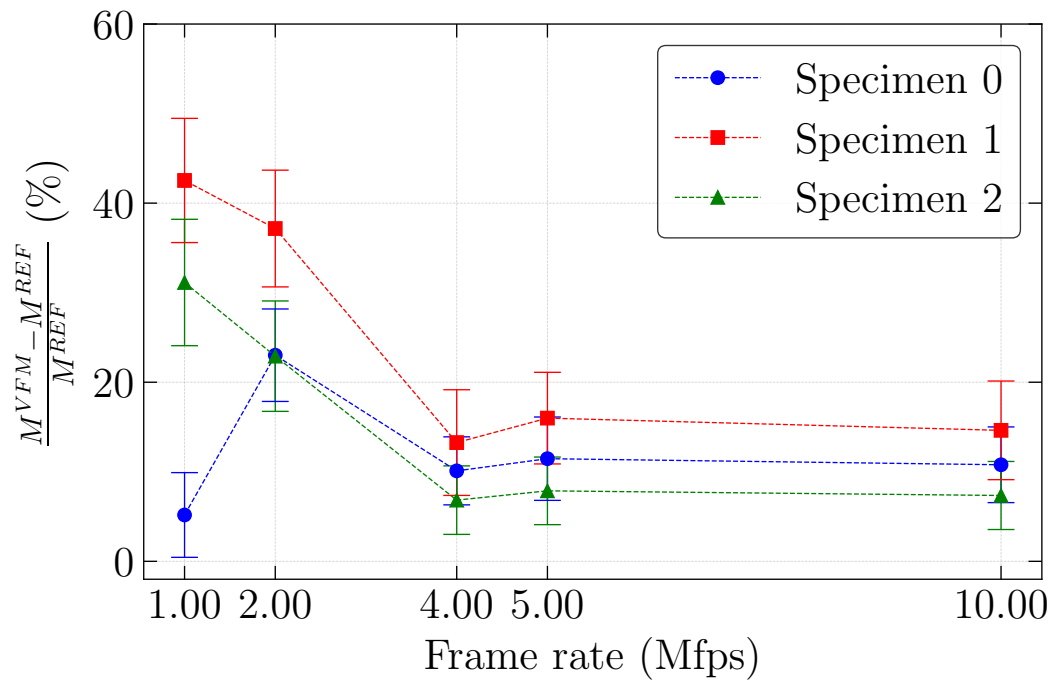

Fig. 14 Identification gap w.r.t reference value on noisy images

occur at the edge root of the concentrator. Moreover, the analysis of the expanded uncertainty on the identification of $M$ enables the discrimination of notched specimen with respect to rectangular and holed specimens. Finally, it is shown that the temporal resolution of the Shimadzu HPV-X® camera is enough to reach temporal convergence, which opens up the way to experimental implementation. 


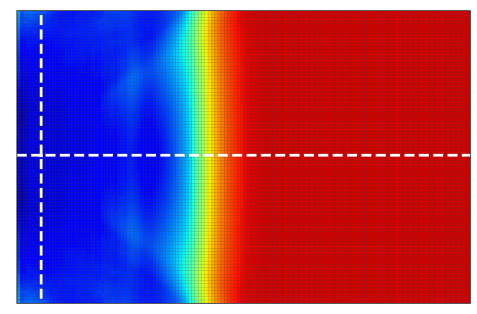

(a)

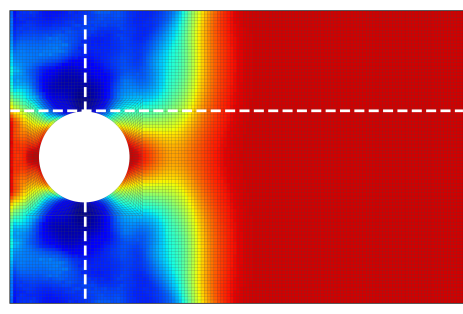

(b)

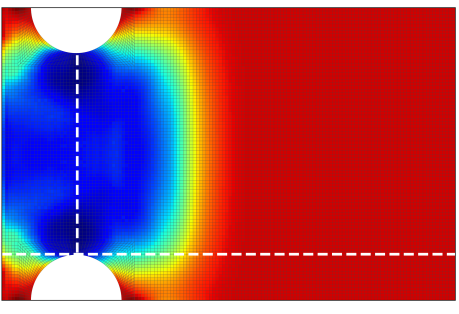

(c)

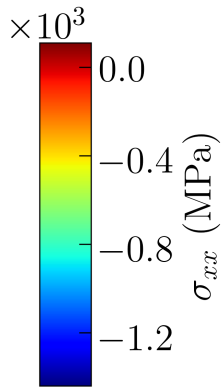

Fig. $15 \sigma_{x x}$ at $\mathrm{t}=6 \mu \mathrm{s}$ - (a) Specimen 0 - (b) Specimen 1 - (b) Specimen 2

\section{Conclusion}

This paper presents a computational approach to design Image-Based Inertial Impact (IBII) tests for the characterization of viscoplasticity at high strain rate. This test has been recently introduced to take advantage of the VFM which enables the identification of material parameters with the sole knowledge of strain and acceleration fields. As this work is seminal for IBII tests and rate-dependent material behaviour characterization, it was attempted to understand IBII test parameters influence on the identification performance. Thus, an FE-based toolchain was proposed to analyze the tests configurations, based on two design criteria: the viscoplastic flow development and the parameters identifiability in realistic conditions, taking into account the measurement toolchain. The current study has led to the following conclusions:

- to increase the strain rate heterogeneity, it is necessary to "machine" holes/notches. However, this has to be done carefully because it may jeopardize material parameter identification if spatial resolution is low;

- the VFM enables the identification of JC viscoplastic parameter $M$ with IBII tests. However, the identification toolchain (including regularization parameters), has to be tuned carefully notably to extract strain and acceleration from noisy displacement maps;

- among the proposed specimens, the notched specimen is less sensitive to noise while guaranteeing identification over a wider viscoplastic spectra than the rectangular and the holed specimen. However, this conclusion is only valid for the impact conditions and the VFM procedure considered in this work,

- simultaneous identification of all JC parameters with the VFM is the next step to obtain all viscoplastic parameters from a single high strain rate test. This may need to resort the sensitivity-based virtual fields proposed in [51] to ensure enough identification stability. This will be explored in the near future.

\section{Acknowledgments}

The authors are grateful to Onera and the Région Hauts-de-France for cofunding this project. Prof. Pierron acknowledges support by EPSRC through grant EP/L026910/1.

\section{References}

1. ASTM E8 - 04, Standard Test Methods for Tension Testing of Metallic Materials, ASTM International, West Conshohocken, PA, 2004. 


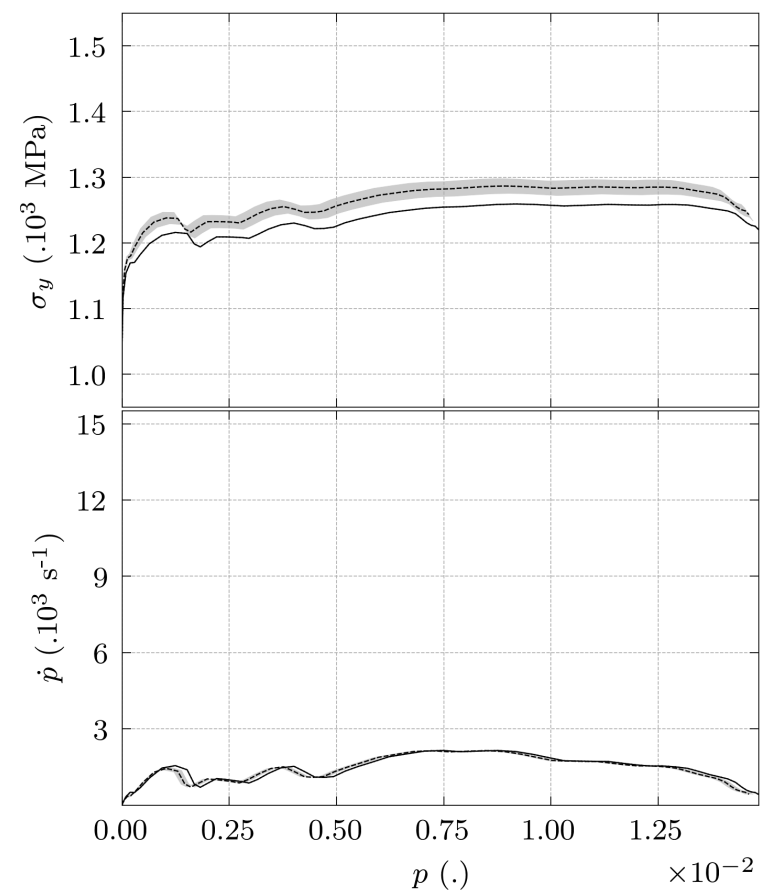

(a)

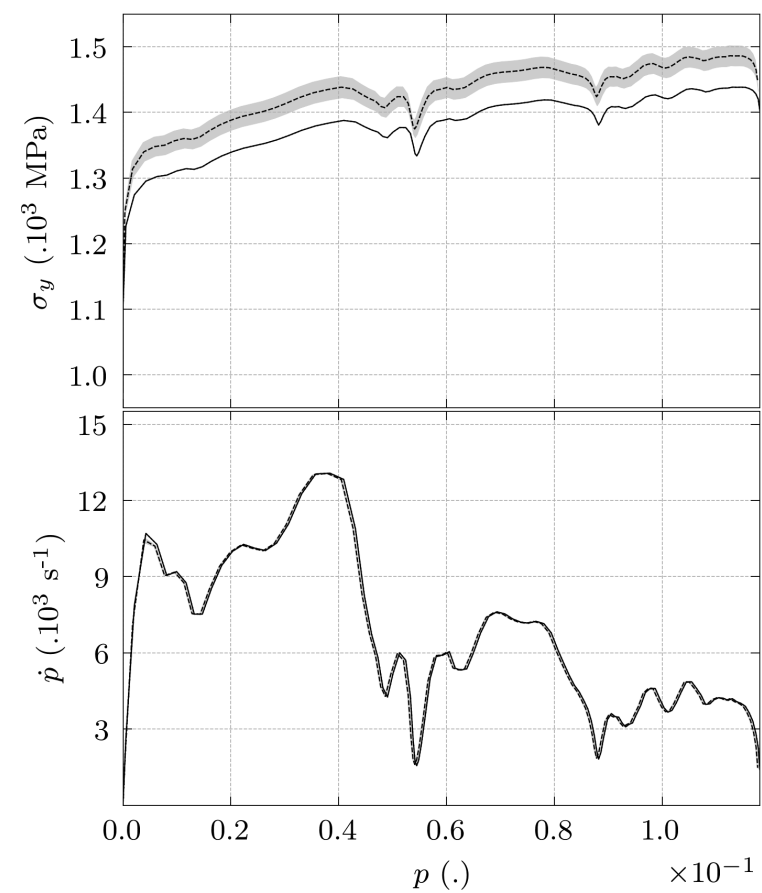

(b)

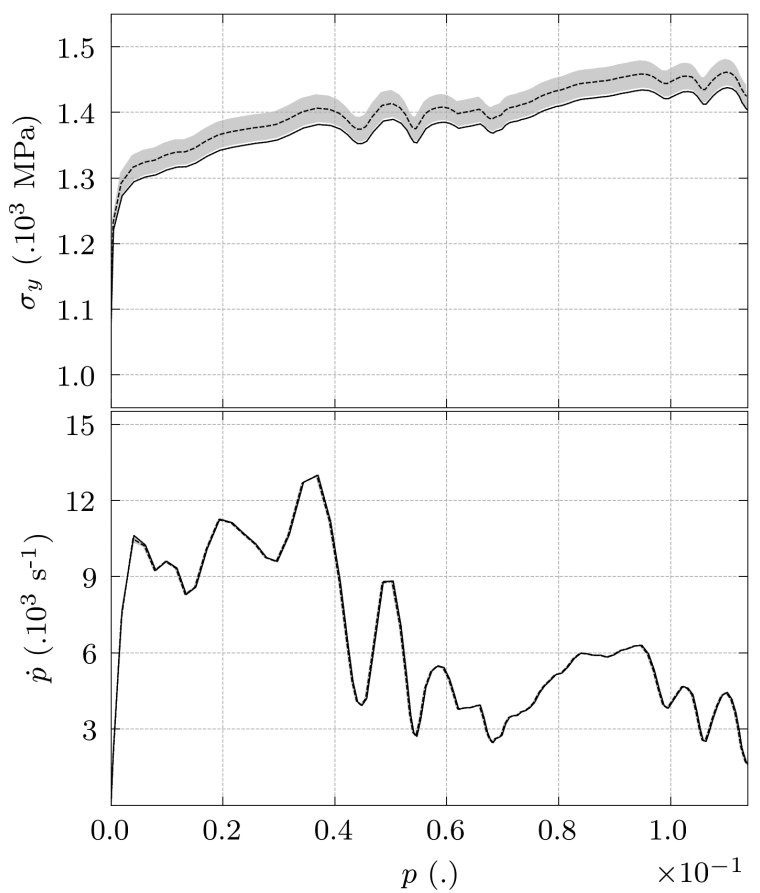

(c)

Fig. $16 \sigma_{x x}$ reconstructed with identified values - (a) Specimen 0 - (b) Specimen 1 - (b) Specimen 2

2. E. Markiewicz, B. Langrand, and D. Notta-Cuvier. A review of characterisation and parameters identification of materials constitutive and damage models: From normalised direct approach to most advanced inverse problem resolution. International Journal of Impact Engineering, 110:371 - 381, 2017.

3. S. Avril, M. Bonnet, AS. Bretelle, M. Grédiac, F. Hild, P. Ienny, F. Latourte, D. Lemosse, S. Pagano, E. Pagnacco, and F. Pierron. Overview of identification methods of mechanical parameters based on full-field measurements. Experimental Mechanics, 48:381-402, 2008.

4. M. Grédiac and F. Hild. Full-Field Measurements and Identification in Solid Mechanics. ISTE. Wiley, 2012. 
5. F. Pierron and M. Grédiac. The Virtual Fields Method: Extracting Constitutive Mechanical Parameters from Full-field Deformation Measurements. Springer New York, 2012.

6. R. Moulart, F. Pierron, S.R. Hallett, and M.R. Wisnom. Full-field strain measurement and identification of composites moduli at high strain rate with the virtual fields method. Experimental Mechanics, 51:509-536, 2011.

7. D. Notta-Cuvier, B. Langrand, E. Markiewicz, F. Lauro, and G. Portemont. Identification of Johnson-Cook's Viscoplastic Model Parameters Using the Virtual Fields Method : Application to Titanium Alloy Ti6Al4V. Strain, 49:22-45, 2013.

8. G. Valeri, B. Koohbor, A. Kidane, and M.A. Sutton. Determining the tensile response of materials at high temperature using DIC and the Virtual Fields Method. Optics and Lasers in Engineering, 91:53-61, 2017.

9. B. Koohbor, A. Kidane, M. A. Sutton, X. Zhao, and S. Mallon. Analysis of dynamic bending test using Ultra High Speed DIC and the virtual fields method. International Journal of Impact Engineering, 110:299 - 310, 2017. Special Issue in honor of Seventy Fifth Birthday of Professor N. K. Gupta.

10. B. Koohbor, A. Kidane, M. A. Sutton, X. Zhao, and S. Mallon. Corrigendum to "Analysis of dynamic bending test using Ultra High Speed DIC and the Virtual Fields Method" [Int. J. Impact Eng. 110 (2017) 299-310]. International Journal of Impact Engineering, 116:127 - 128, 2018.

11. F. Pierron, M. A. Sutton, and V. Tiwari. Ultra High Speed DIC and virtual fields method analysis of a three point bending impact test on a aluminium bar. Experimental Mechanics, 51:537-563, 2010.

12. F. Pierron, H. Zhu, and C. Siviour. Beyond Hopkinson's bar. Philosophical Transactions of the Royal Society of London A: Mathematical, Physical and Engineering Sciences, 372, 2014.

13. S. Dreuilhe. Development of novel inertial tests for the identification of non-linear material model parameters at high strain rates. PhD thesis, University of Southampton, 2017.

14. J. Lemaitre and J.L. Chaboche. Mechanics of Solid Materials. Cambridge University Press, 1994.

15. F. J. Zerilli and R. W. Armstrong. Dislocation mechanics based constitutive relations for material dynamics calculations. Journal of Applied Physics, 61(5):1816-1825, 1987.

16. A. Rusinek and J.R Klepaczko. Shear testing of a sheet steel at wide range of strain rates and a constitutive relation with strain-rate and temperature dependence of the flow stress. International Journal of Plasticity, 17(1):87 - $115,2001$.

17. G.R. Cowper and P.S. Symonds. Strain-hardening and Strain-rate Effects in the Impact Loading of Cantilever Beams. Technical report (Brown University. Division of Applied Mathematics). Division of Applied Mathematics, Brown University, 1957.

18. G.R. Johnson and W.H. Cook. A constitutive model and data for metals subjected to large strains, high strain rates and high temperatures. Proc. 7th International Symposium on Balistics, The Hague, The Netherlands, pages 541-547, 1983.

19. Norman Jones. Structural Impact. Cambridge University Press, 1990.

20. T. J. Holmquist and G. R. Johnson. Determination of constants and comparison of results for various constitutive models. J. Phys. IV France, 01:C3-853-C3-860, 1991.

21. T. Borvik, M. Langseth, O.S. Hopperstad, and K.A. Malo. Ballistic penetration of steel plates. International Journal of Impact Engineering, 22:855-886, 1999.

22. A. K. Gupta, H. N. Krishnamurthy, P. Puranik, S. K. Singh, and A. Balu. An exponential strain dependent Rusinek-Klepaczko model for flow stress prediction in austenitic stainless steel 304 at elevated temperatures. Journal of Materials Research and Technology, 3(4):370 - 377, 2014.

23. M.A. Iqbal, K. Senthil, P. Sharma, and N.K. Gupta. An investigation of the constitutive behavior of Armox 500T steel and armor piercing incendiary projectile material. International Journal of Impact Engineering, 96:146 - 164, 2016.

24. H.W. Meyer Jr and D.S. Kleponis. Modeling the high strain rate behavior of titanium undergoing ballistic impact and penetration. International Journal of Impact Engineering, 26:509-521, 2001.

25. J. Peirs, P. Verleysen, W. Van Paepegem, and J. Degrieck. Determining the stress-strain behaviour at large strains from high strain rate tensile and shear experiments. International Journal of Impact Engineering, 38:406-415, 2011. 
26. W. Mocko and A. Brodecki. Application of optical field analysis of tensile tests for calibration of the Rusinek-Klepaczko constitutive relation of Ti6Al4V titanium alloy. Materials and Design, 88:320-330, 2015.

27. B. Hopkinson. A method of measuring the pressure produced in the detonation of high explosives of by the impact of bullets. Philosophical Transactions of the Royal Society A, 213:437-456, 1914.

28. T. Nicholas. Tensile testing of materials at high rates of strain. Experimental Mechanics, 21(5):177-185, May 1981.

29. S. Avril, F. Pierron, M. A. Sutton, and J. Yan. Identification of elasto-visco-plastic parameters and characterization of Lüders behavior using digital image correlation and the virtual fields method. Mechanics of Materials, 40:729-742, 2008.

30. F. Pierron, S.Avril, and V. T. Tran. Extension of the virtual fields method to elasto-plastic material identification with cyclic loads and kinematic hardening. International Journal of Solids and Structures, 47:2993-3010, 2010.

31. D. Notta-Cuvier, B. Langrand, F. Lauro, and E. Markiewicz. An innovative procedure for characterising a coupled elastoplastic damage model of behaviour using the virtual fields method. International Journal of Solids and Structures, 69-70:415-427, 2015 .

32. F. Pierron and P. Forquin. Ultra-high-speed full-field deformation measurements on concrete spalling specimens and stiffness identification with the virtual fields method. Strain, 48:388-405, 2012.

33. S.-H. Yoon, M. Winters, and C.R. Siviour. High Strain-Rate Tensile Characterization of EPDM Rubber Using Nonequilibrium Loading and the Virtual Fields Method. Experimental Mechanics, 56:25-25, 2016.

34. L. Fletcher and F. Pierron. An Image-Based Inertial Impact (IBII) Test for Tungsten Carbide Cermets. Journal of Dynamic Behavior of Materials, Aug 2018.

35. J. Van Blitterswyk, L. Fletcher, and F. Pierron. Image-Based Inertial Impact Test for Composite Interlaminar Tensile Properties. Journal of Dynamic Behavior of Materials, Sep 2018. Available online.

36. G. Le Louëdec, F. Pierron, M. A. Sutton, C. Siviour, and A. P. Reynolds. Identification of the Dynamic Properties of Al 5456 FSW Welds Using the Virtual Fields Method. Journal of Dynamic Behavior of Materials, 1(2):176-190, Jun 2015.

37. M. Grédiac, B. Blaysat, and F. Sur. A Critical Comparison of Some Metrological Parameters Characterizing Local Digital Image Correlation and Grid Method. Experimental Mechanics, 57(6):871-903, 2017.

38. M. Grédiac, F. Sur, and B. Blaysat. The Grid Method for In-plane Displacement and Strain Measurement: A Review and Analysis. Strain, 52(3):205-243, 2016.

39. H. Abdul-Rahman, M. Gdeisat, D. Burton, and M. Lalor. Fast three-dimensional phase-unwrapping algorithm based on sorting by reliability following a non-continuous path. In Proc. SPIE, volume 5856, pages 32-40, 2005.

40. P. Holoborodko. (n.d) Smooth noise-robust differentiators. Retrieved from http://www.holoborodko.com/pavel/numericalmethods/noise-robust-smoothing-filter/.

41. M. Bornert, F. Brémand, P. Doumalin, J.-C. Dupré, M. Fazzini, M. Grédiac, F. Hild, S. Mistou, J. Molimard, J.-J. Orteu, L. Robert, Y. Surrel, P. Vacher, and B. Wattrisse. Assessment of Digital Image Correlation Measurement Errors: Methodology and Results. Experimental Mechanics, 49(3):353-370, Jun 2009.

42. M. Rossi and F. Pierron. On the use of simulated experiments in designing tests for material characterization from full-field measurements. International Journal of Solids and Structures, 49(3-4):420 - 435, 2012.

43. M. Rossi, P. Lava, F. Pierron, D. Debruyne, and M. Sasso. Effect of DIC Spatial Resolution, Noise and Interpolation Error on Identification Results with the VFM. Strain, 51:206-222, 2015.

44. B. Lukić, D. Saletti, and P. Forquin. Use of simulated experiments for material characterization of brittle materials subjected to high strain rate dynamic tension. Philosophical Transactions of the Royal Society of London A: Mathematical, Physical and Engineering Sciences, 375(2085), 2016.

45. J. J. Orteu, D. Garcia, L. Robert, and F. Bugarin. A speckle texture image generator. Proc. SPIE, $6341,2006$.

46. R. Balcaen, L. Wittevrongel, P. L. Reu, P. Lava, and D. Debruyne. Stereo-DIC Calibration and Speckle Image Generator Based on FE Formulations. Experimental Mechanics, 57(5):703-718, 2017.

47. Cast3M@. (n.d) http://www-cast3m.cea.fr. 
48. Europlexus Team. Europlexus: a computer program for the finite element simulation of fluid-structure systems under transient dynamic loading - User's manual, 2017.

49. J. C. Simo and T. J. R. Hughes. Computational Inelasticity. Springer, 1998.

50. E.A. de Souza Neto, D. Peric, and D.R.J. Owen. Computational Methods for Plasticity: Theory and Applications. Wiley, 2008.

51. A. Marek, F. M. Davis, and F. Pierron. Sensitivity-based virtual fields for the non-linear virtual fields method. Computational Mechanics, 60(3):409-431, Sep 2017. 